hep-ph/yymmxxx

UWThPh-1998-60

HEPHY-PUB 702/98

FTUV/98-96

IFIC $/ 98-98$

July 6,2021

\title{
More on higher order decays of the lighter top squark
}

\author{
W. Porod* \\ Institut für Theoretische Physik, Universität Wien, A-1090 Vienna, Austria \\ and \\ Departamento de Física Teórica, IFIC-CSIC, Universidad de Valencia, \\ Burjassot, Valencia 46100, Spain
}

\begin{abstract}
We discuss the three-body decays $\tilde{t}_{1} \rightarrow W^{+} b \tilde{\chi}_{1}^{0}, \tilde{t}_{1} \rightarrow H^{+} b \tilde{\chi}_{1}^{0}, \tilde{t}_{1} \rightarrow b \tilde{l}_{i}^{+} \nu_{l}$, and $\tilde{t}_{1} \rightarrow b \tilde{\nu}_{l} l^{+}(l=e, \mu, \tau)$ of the lighter top squark $\tilde{t}_{1}$ within the minimal supersymmetric standard model. We give the complete analytical formulas for the decay widths and present a numerical study in view of an upgraded Tevatron, the CERN LHC, and a future lepton collider demonstrating the importance of these decay modes.
\end{abstract}

14.80.Ly, 11.30.Pb, 12.60.Jv

Typeset using REVTEX

*Electronic address: porod@ap.univie.ac.at 


\section{INTRODUCTION}

The search for supersymmetry (SUSY) [1, 2] plays an important rôle in the experimental program at the colliders LEP2 and Tevatron. It will be even more important at future colliders, e.g. an upgraded Tevatron, LHC, an $e^{+} e^{-}$linear collider or a $\mu^{+} \mu^{-}$collider. Therefore many phenomenological studies have been carried out in recent years (see e.g. [3 6] and references therein).

Within the supersymmetric extensions of the standard model (SM) the minimal supersymmetric standard model (MSSM) [2,7] is the most investigated one. The MSSM implies that every SM fermion has two spin 0 partners called sfermions $\tilde{f}_{L}$ and $\tilde{f}_{R}$. In general sfermions decay according to $\tilde{f}_{k} \rightarrow f \tilde{\chi}_{i}^{0}, f^{\prime} \tilde{\chi}_{j}^{ \pm}$where $\tilde{\chi}_{i}^{0}$ and $\tilde{\chi}_{j}^{ \pm}$denote neutralinos and charginos, respectively. Here we assume that the lightest neutralino is the lightest supersymmetric particle (LSP).

Owing to large Yukawa couplings the sfermions of the third generation have a quite different phenomenology compared to those of the first two generations (see e.g. [8] and references therein). The large Yukawa couplings imply a large mixing between $\tilde{f}_{L}$ and $\tilde{f}_{R}$ and large couplings to the higgsino components of neutralinos and charginos. This is in particular the case for the lighter top squark $\tilde{t}_{1}$ because of the large top quark mass [9]. The large top quark mass also implies the existence of scenarios where all two-body decay modes of $\tilde{t}_{1}$ (e.g. $\tilde{t}_{1} \rightarrow t \tilde{\chi}_{i}^{0}, b \tilde{\chi}_{j}^{+}, t \tilde{g}$ ) are kinematically forbidden at tree-level. In these scenarios higher order decays of $\tilde{t}_{1}$ become relevant: [10]11]:

$$
\begin{aligned}
& \tilde{t}_{1} \rightarrow c \tilde{\chi}_{1,2}^{0} \\
& \tilde{t}_{1} \rightarrow W^{+} b \tilde{\chi}_{1}^{0} \\
& \tilde{t}_{1} \rightarrow H^{+} b \tilde{\chi}_{1}^{0} \\
& \tilde{t}_{1} \rightarrow b \tilde{l}_{i}^{+} \nu_{l} \\
& \tilde{t}_{1} \rightarrow b \tilde{\nu}_{l} l^{+},
\end{aligned}
$$

where $l$ denotes $e, \mu, \tau$.

In [10] it has been shown that decays into sleptons are dominating over the decays into $c \tilde{\chi}_{1,2}^{0}$ if they are kinematically allowed. However, they have used the approximation: $m_{b}=0, h_{b}=h_{l}=0(l=e, \mu, \tau), m_{\tilde{t}_{1}} \ll m_{\tilde{\chi}_{1}^{+}} \ll m_{\tilde{\chi}_{2}^{+}}$. In [11 it has been shown that for small $\tan \beta$ the decay $\tilde{t}_{1} \rightarrow W^{+} b \tilde{\chi}_{1}^{0}$ in general dominates over $\tilde{t}_{1} \rightarrow c \tilde{\chi}_{1,2}^{0}$ whereas for large $\tan \beta$ their branching ratios can be of comparable size. In this paper we present the complete formulas for the three-body decays which are so far missing in the literature. We also perform a numerical analysis for the mass range of an upgraded Tevatron, the LHC, and a future lepton collider including the possibility that all of the above decay channels are simultaneously open. In particular it turns out that the inclusion of the bottom and tau Yukawa couplings $h_{b}$ and $h_{\tau}$ is important.

This paper is organized as follows: In Sect. II we fix our notation and give the analytical expressions for the decay amplitudes together with the relevant parts of the MSSM Lagrangian. In Sect. III we present our numerical results for the branching ratios of the three-body decays in scenarios accessible either at the Tevatron run II, LHC, or a future lepton collider. Our conclusions are drawn in Sect. IV. The analytical formulas for the squared amplitudes are listed in Appendix A, and Appendix B gives the various couplings. 


\section{FORMULAS FOR THE DECAY WIDTHS}

In this section we fix our notation and we give the Lagrangian relevant for the calculation of the decay widths. Moreover, we present the analytical formulas for the matrix elements and generic formulas for the decay widths. The complete formulas for the latter are rather lengthy and are listed in Appendix A.

The parameters relevant for the following discussion are $M^{\prime}, M, m_{A^{0}}, \mu, \tan \beta, M_{\tilde{D}_{i}}, M_{\tilde{Q}_{i}}$, $M_{\tilde{U}_{i}}, A_{d_{i}}$, and $A_{u_{i}} . M^{\prime}$ and $M$ are the $U(1)$ and $S U(2)$ gaugino masses, for which we assume the GUT relation $M^{\prime}=5 / 3 \tan ^{2} \theta_{W} M . \mu$ is the parameter of the Higgs superpotential, $m_{A^{0}}$ the mass of the pseudoscalar Higgs boson, and $\tan \beta=v_{2} / v_{1}$ where $v_{i}$ denotes the vacuum expectation value of the Higgs doublet $H_{i} . \quad M_{\tilde{D}_{i}}, M_{\tilde{Q}_{i}}$ and $M_{\tilde{U}_{i}}$ are soft SUSY breaking masses for the squarks, $A_{d_{i}}$ and $A_{u_{i}}$ are trilinear Higgs-squark couplings, and $i=1,2,3$ is the generation index.

The mass matrix for sfermions in the $\left(\tilde{f}_{L}, \tilde{f}_{R}\right)$ basis has the following form [8]:

$$
\mathcal{M}_{\tilde{f}_{i}}^{2}=\left(\begin{array}{ll}
m_{\tilde{f}_{L i}}^{2} & a_{f_{i}} m_{f_{i}} \\
a_{f_{i}} m_{f_{i}} & m_{\tilde{f}_{R i}}^{2}
\end{array}\right)
$$

with

$$
\begin{aligned}
& m_{\tilde{u}_{L i}}^{2}=M_{\tilde{Q}_{i}}^{2}+m_{u_{i}}^{2}+m_{Z}^{2} \cos 2 \beta\left(\frac{1}{2}-\frac{2}{3} \sin ^{2} \theta_{W}\right), \\
& m_{\tilde{u}_{R i}}^{2}=M_{\tilde{U}_{i}}^{2}+m_{u_{i}}^{2}+\frac{2}{3} m_{Z}^{2} \cos 2 \beta \sin ^{2} \theta_{W} \\
& m_{\tilde{d}_{L i}}^{2}=M_{\tilde{Q}_{i}}^{2}+m_{d_{i}}^{2}-m_{Z}^{2} \cos 2 \beta\left(\frac{1}{2}-\frac{1}{3} \sin ^{2} \theta_{W}\right), \\
& m_{\tilde{d}_{R i}}^{2}=M_{\tilde{D}_{i}}^{2}+m_{d_{i}}^{2}-\frac{1}{3} m_{Z}^{2} \cos 2 \beta \sin ^{2} \theta_{W} \\
& m_{\tilde{l}_{L i}}^{2}=M_{\tilde{L}_{i}}^{2}+m_{l_{i}}^{2}-m_{Z}^{2} \cos 2 \beta\left(\frac{1}{2}-\sin ^{2} \theta_{W}\right), \\
& m_{\tilde{l}_{R i}}^{2}=M_{\tilde{E}_{i}}^{2}+m_{l_{i}}^{2}-m_{Z}^{2} \cos 2 \beta \sin ^{2} \theta_{W}
\end{aligned}
$$

and

$$
\begin{aligned}
a_{u_{i}} m_{u_{i}} & =m_{u_{i}}\left(A_{u_{i}}-\mu \cot \beta\right), \\
a_{d_{i}} m_{d_{i}} & =m_{d_{i}}\left(A_{d_{i}}-\mu \tan \beta\right), \\
a_{l_{i}} m_{l_{i}} & =m_{l_{i}}\left(A_{l_{i}}-\mu \tan \beta\right),
\end{aligned}
$$

where $i$ is a generation index $\left(u_{i}=u, c, t ; d_{i}=d, s, b ; l_{i}=e, \nu, \tau\right)$ which will be suppressed in the following. The mass eigenstates $\tilde{f}_{1}$ and $\tilde{f}_{2}$ are related to $\tilde{f}_{L}$ and $\tilde{f}_{R}$ by:

$$
\left(\begin{array}{c}
\tilde{f}_{1} \\
\tilde{f}_{2}
\end{array}\right)=\left(\begin{array}{cc}
\cos \theta_{\tilde{f}} & \sin \theta_{\tilde{f}} \\
-\sin \theta_{\tilde{f}} & \cos \theta_{\tilde{f}}
\end{array}\right)\left(\begin{array}{c}
\tilde{f}_{L} \\
\tilde{f}_{R}
\end{array}\right)=\mathcal{R}^{\tilde{f}}\left(\begin{array}{c}
\tilde{f}_{L} \\
\tilde{f}_{R}
\end{array}\right)
$$

with the eigenvalues

$$
m_{\tilde{f}_{1,2}}^{2}=\frac{1}{2}\left(m_{\tilde{f}_{L}}^{2}+m_{\tilde{f}_{R}}^{2}\right) \mp \frac{1}{2} \sqrt{\left(m_{\tilde{f}_{L}}^{2}-m_{\tilde{f}_{R}}^{2}\right)^{2}+4 a_{f}^{2} m_{f}^{2}} .
$$

The mixing angle $\theta_{f}$ is given by

$$
\cos \theta_{\tilde{f}}=\frac{-a_{f} m_{f}}{\sqrt{\left(m_{\tilde{f}_{L}}^{2}-m_{\tilde{f}_{1}}^{2}\right)^{2}+a_{f}^{2} m_{f}^{2}}}, \quad \sin \theta_{\tilde{f}}=\sqrt{\frac{\left(m_{\tilde{f}_{L}}^{2}-m_{\tilde{f}_{1}}^{2}\right)^{2}}{\left(m_{\tilde{f}_{L}}^{2}-m_{\tilde{f}_{1}}^{2}\right)^{2}+a_{f}^{2} m_{f}^{2}}} .
$$


The sneutrino mass is given by:

$$
m_{\tilde{\nu}_{i}}^{2}=M_{\tilde{L}_{i}}^{2}+\frac{1}{2} m_{Z}^{2} \cos 2 \beta
$$

The part of the Lagrangian, which is needed for the calculation of the three-body decay widths, is given by:

$$
\begin{aligned}
& \mathcal{L}_{I}=g \sum_{j=1,2} \bar{b}\left(k_{1 j}^{\tilde{t}} P_{L}+l_{1 j}^{\tilde{t}} P_{R}\right) \tilde{\chi}_{j}^{+} \tilde{t}_{1}+g \sum_{\substack{j, n=1,2 \\
f=e, \mu, \tau}} \overline{\nu_{f}}\left(k_{n j}^{\tilde{f}} P_{L}+l_{n j}^{\tilde{f}} P_{R}\right) \tilde{\chi}_{j}^{-} \tilde{f}_{n} \\
& +g \sum_{\substack{j=1,2 \\
f=e, \mu, \tau}} \overline{\tilde{\chi}_{j}^{+}}\left(l_{j}^{\tilde{\nu}_{f}} P_{L}\right) f \tilde{\nu}_{f}^{\dagger}+g \sum_{\substack{k=1,2 \\
f=b, t}} \bar{f}\left(b_{k 1}^{f} P_{L}+a_{k 1}^{f} P_{R}\right) \tilde{\chi}_{1}^{0} \tilde{f}_{k} \\
& -g W_{\mu}^{-} \sum_{j=1,2}^{2} \overline{\tilde{\chi}_{1}^{0}}\left(O_{j 1}^{L} P_{L}+O_{j 1}^{R} P_{R}\right) \gamma^{\mu} \tilde{\chi}_{j}^{+}-i g W_{\mu}^{-} \sum_{j=1,2}^{2} A_{\tilde{t}_{1} \tilde{b}_{j}}^{W} \tilde{b}_{j}^{\dagger} \stackrel{\leftrightarrow}{\partial_{\mu}} \tilde{t}_{1} \\
& -\frac{g}{\sqrt{2}} W_{\mu}^{-\bar{b}} \gamma^{\mu} P_{L} t-g H^{-} \sum_{j=1,2}^{2} \overline{\tilde{\chi}_{1}^{0}}\left(Q_{1 j}^{R \prime} P_{L}+Q_{1 i}^{L \prime} P_{R}\right) \tilde{\chi}_{j}^{+}-g H^{-} \sum_{j=1,2}^{2} C_{\tilde{t}_{1} \tilde{b}_{j}}^{H} \tilde{t}_{1} \tilde{b}_{j}^{\dagger} \\
& +\frac{g}{\sqrt{2} m_{W}} H^{-} \bar{b}\left(m_{b} \tan \beta P_{L}+m_{t} \cot \beta P_{R}\right) t
\end{aligned}
$$

where $P_{R, L}=\left(1 \pm \gamma_{5}\right) / 2$. The various couplings are given in Appendix $\mathrm{B}$.

The formula for the decay width $\Gamma\left(\tilde{t}_{1} \rightarrow W^{+} b \tilde{\chi}_{1}^{0}\right)$ has already been given in [1]. Therefore, we give only the corresponding matrix element $M_{\tilde{t}_{1} \rightarrow W^{+} b \tilde{\chi}_{1}^{0}}$ :

$$
\begin{aligned}
M_{\tilde{t}_{1} \rightarrow W^{+} b \tilde{\chi}_{1}^{0}}= & -\frac{g^{2}}{\sqrt{2}} \sum_{j=1}^{2} A_{\tilde{t}_{1} \tilde{b}_{j}}^{W} \frac{\left(p_{\tilde{t}}+p_{\tilde{b}_{j}}\right)^{\mu}}{p_{\tilde{b}_{j}}^{2}-m_{\tilde{b}_{j}}^{2}-i m_{\tilde{b}_{j}} \Gamma_{\tilde{b}_{j}}} \bar{u}\left(p_{b}\right)\left[b_{j 1}^{\tilde{b}} P_{L}+a_{j 1}^{\tilde{b}} P_{R}\right] v\left(p_{\tilde{\chi}_{1}^{0}}\right) \epsilon_{\mu}\left(p_{W}\right) \\
& +g^{2} \sum_{j=1}^{2} \bar{u}\left(p_{b}\right)\left[l_{1 j}^{\tilde{t}} P_{R}+k_{1 j}^{\tilde{t}} P_{L}\right] \frac{\not p_{\tilde{\chi}_{j}^{+}}-m_{\tilde{\chi}_{j}^{+}}}{p_{\tilde{\chi}_{j}^{+}}^{2}-m_{\tilde{\chi}_{j}^{+}}^{2}-i m_{\tilde{\chi}_{j}^{+}} \Gamma_{\tilde{\chi}_{j}^{+}}} \\
& *\left[O_{1 j}^{L \prime} P_{L}+O_{1 j}^{R \prime} P_{R}\right] \gamma^{\mu} v\left(p_{\tilde{\chi}_{1}^{0}}\right) \epsilon_{\mu}\left(p_{W}\right) \\
& -\frac{g^{2}}{\sqrt{2}} \bar{u}\left(p_{b}\right) \gamma^{\mu} P_{L} \frac{\not p_{t}+m_{t}}{p_{t}^{2}-m_{t}^{2}-i m_{t} \Gamma_{t}}\left[b_{11}^{\tilde{t}} P_{L}+a_{11}^{\tilde{t}} P_{R}\right] v\left(p_{\tilde{\chi}_{1}^{0}}\right) \epsilon_{\mu}\left(p_{W}\right)
\end{aligned}
$$

In 111 also the formula for $\Gamma\left(\tilde{t}_{1} \rightarrow c \tilde{\chi}_{1}^{0}\right)$ 《10 has been rewritten in the notation used here.

In Fig. 1 we show the Feynman diagrams for the decay $\tilde{t}_{1} \rightarrow H^{+} b \tilde{\chi}_{1}^{0}$. The matrix element $M_{\tilde{t}_{1} \rightarrow H^{+} b \tilde{\chi}_{1}^{0}}$ for this decay is given by:

$$
\begin{aligned}
M_{\tilde{t}_{1} \rightarrow H^{+} b \tilde{\chi}_{1}^{0}}= & -g^{2} \sum_{j=1}^{2} C_{\tilde{t}_{1} \tilde{b}_{j}}^{H} \frac{\bar{u}\left(p_{b}\right)\left[b_{j 1}^{\tilde{b}} P_{L}+a_{j 1}^{\tilde{b}} P_{R}\right] v\left(p_{\tilde{\chi}_{1}^{0}}\right)}{p_{\tilde{b}_{j}}^{2}-m_{\tilde{b}_{j}}^{2}-i m_{\tilde{b}_{j}} \Gamma_{\tilde{b}_{j}}} \\
& -g^{2} \sum_{j=1}^{2} \frac{\bar{u}\left(p_{b}\right)\left[l_{1 j}^{\tilde{t}} P_{R}+k_{1 j}^{\tilde{t}} P_{L}\right]\left[\not p_{\tilde{\chi}_{j}^{+}}-m_{\tilde{\chi}_{j}^{+}}\right]\left[Q_{1 j}^{L \prime}{ }^{\prime} P_{L}+Q_{1 j}^{R \prime} P_{R}\right] v\left(p_{\tilde{\chi}_{1}^{0}}\right)}{p_{\tilde{\chi}_{j}^{+}}^{2}-m_{\tilde{\chi}_{j}^{+}}^{2}-i m_{\tilde{\chi}_{j}{ }^{+}} \Gamma_{\tilde{\chi}_{j}^{+}}} \\
& +\frac{g^{2}}{\sqrt{2} m_{W}} \frac{\bar{u}\left(p_{b}\right)\left[m_{b} \tan \beta P_{L}+m_{t} \cot \beta P_{R}\right]\left[\not p_{t}+m_{t}\right]\left[b_{11}^{\tilde{t}} P_{L}+a_{11}^{\tilde{t}} P_{R}\right] v\left(p_{\tilde{\chi}_{1}^{0}}\right)}{p_{t}^{2}-m_{t}^{2}-i m_{t} \Gamma_{t}}
\end{aligned}
$$


The decay width is given by

$$
\begin{gathered}
\Gamma\left(\tilde{t}_{1} \rightarrow H^{+} b \tilde{\chi}_{1}^{0}\right)= \\
=\frac{\alpha^{2}}{16 \pi m_{\tilde{t}_{1}}^{3} \sin ^{4} \theta_{W}} \int_{\left(m_{b}+m_{\tilde{\chi}_{1}^{0}}\right)^{2}}^{\left(m_{\tilde{t}_{1}}-m_{H^{+}}\right)^{2}} d s\left(G_{\tilde{\chi}^{+} \tilde{\chi}^{+}}+G_{\tilde{\chi}^{+} t}+G_{\tilde{\chi}^{+} \tilde{b}}+G_{t t}+G_{t \tilde{b}}+G_{\tilde{b} \tilde{b}}\right) .
\end{gathered}
$$

with $G_{i j}$ given in Appendix A.

Alternatively $\tilde{t}_{1}$ can decay into sleptons: $\tilde{t}_{1} \rightarrow b \tilde{\nu}_{e} e^{+}, b \tilde{\nu}_{\mu} \mu^{+}, \tilde{t}_{1} \rightarrow b \tilde{e}_{L}^{+} \nu_{e}, b \tilde{\mu}_{L}^{+} \nu_{\mu}$, $\tilde{t}_{1} \rightarrow b \tilde{\nu}_{\tau} \tau^{+}$, and $\tilde{t}_{1} \rightarrow b \tilde{\tau}_{1,2}^{+} \nu_{\tau}$. These decays are mediated through virtual charginos. The Feynman graphs are similar to the second one in Fig. 1, where one has to replace the Higgs boson by a slepton and the neutralino by the corresponding lepton. Note, that the decays into $\tilde{e}_{R}$ and $\tilde{\mu}_{R}$ are negligible because their couplings to the charginos are proportional to $m_{e} / m_{W}$ and $m_{\mu} / m_{W}$, respectively. In the case of decays into sneutrinos and leptons the matrix elements $M_{\tilde{t}_{1} \rightarrow b l^{+} \tilde{\nu}_{l}}$ have the generic form:

$$
M_{\tilde{t}_{1} \rightarrow b l^{+} \tilde{\nu}_{l}}=g^{2} \sum_{j=1}^{2} \frac{\bar{u}\left(p_{b}\right)\left[l_{1 j}^{\tilde{t}} P_{R}+k_{1 j}^{\tilde{t}} P_{L}\right]\left[\not p_{\tilde{\chi}_{j}^{+}}-m_{\tilde{\chi}_{j}^{+}}\right]\left[l_{j}^{\tilde{\nu}_{l}} P_{R}+k_{j}^{\tilde{\nu}_{l}} P_{L}\right] v\left(p_{l}\right)}{p_{\tilde{\chi}_{j}^{+}}^{2}-m_{\tilde{\chi}_{j}^{+}}^{2}-i m_{\tilde{\chi}_{j}^{+}} \Gamma_{\tilde{\chi}_{j}^{+}}},
$$

whereas for the decays into sleptons and neutrinos we get:

$$
M_{\tilde{t}_{1} \rightarrow b \nu_{l} \tilde{l}_{k}^{+}}=g^{2} \sum_{j=1}^{2} \frac{\bar{u}\left(p_{b}\right)\left[l_{1 j}^{\tilde{t}} P_{R}+k_{1 j}^{\tilde{t}} P_{L}\right]\left[\not p_{\tilde{\chi}_{j}^{+}}-m_{\tilde{\chi}_{j}^{+}}\right]\left[l_{k j}^{\tilde{l}} P_{L}\right] v\left(p_{\nu_{l}}\right)}{p_{\tilde{\chi}_{j}^{+}}^{2}-m_{\tilde{\chi}_{j}^{+}}^{2}-i m_{\tilde{\chi}_{j}^{+}} \Gamma_{\tilde{\chi}_{j}^{+}}} .
$$

In both cases the decay width is given by

$$
\Gamma\left(\tilde{t}_{1} \rightarrow b \tilde{l} l^{\prime}\right)=\frac{\alpha^{2}}{16 \pi m_{\tilde{t}_{1}}^{3} \sin ^{4} \theta_{W}} \int_{\left(m_{l^{\prime}}+m_{\tilde{l}}\right)^{2}}^{\left(m_{\tilde{t}_{1}}-m_{b}\right)^{2}} d s W_{l^{\prime}}(s) \sum_{i=1}^{3}\left(\sum_{j=1}^{5} c_{i j} s^{(j-4)}\right) D_{i}(s) .
$$

The explicit expressions for $W_{l^{\prime} \tilde{l}}, c_{i j}$, and $D_{i}(s)$ are given in Appendix A.

\section{NUMERICAL RESULTS}

In this section we present our numerical results for the branching ratios of the higher order decays of $\tilde{t}_{1}$. Here we consider scenarios where all two-body decays at tree-level are kinematically forbidden.

We have fixed the parameters as in [11] to avoid colour breaking minimas: we have used $m_{\tilde{t}_{1}}, \cos \theta_{\tilde{t}}, \tan \beta$, and $\mu$ as input parameters in the top squark sector. For the sbottom (stau) sector we have fixed $M_{\tilde{Q}}, M_{\tilde{D}}$ and $A_{b}\left(M_{\tilde{E}}, M_{\tilde{L}}\right.$, and $\left.A_{\tau}\right)$ as input parameters. For simplicity, we assume that the soft SUSY breaking parameters are equal for all generations. Note, that due to $S U(2)$ invariance $M_{\tilde{Q}}$ appears in both up- and down-type squark mass matrices. In the sbottom (stau) sector the physical quantities $m_{\tilde{b}_{1}}, m_{\tilde{b}_{2}}$, and $\cos \theta_{\tilde{b}}\left(m_{\tilde{\tau}_{1}}\right.$, $m_{\tilde{\tau}_{2}}$, and $\left.\cos \theta_{\tilde{\tau}}\right)$ obviously change with $\mu$ and $\tan \beta$. 
In Fig. 2(a) and (b) we show the branching ratios of $\tilde{t}_{1}$ as a function of $\cos \theta_{\tilde{t}}$. We have restricted the $\cos \theta_{\tilde{t}}$ range such that $\left|A_{t}\right| \leq 1 \mathrm{TeV}$ to avoid color/charge breaking minima. The parameters and physical quantities are given in Tab. $\mathbb{\square}$. The slepton parameters have been chosen such that the sum of the masses of the final state particles are $215 \pm 5 \mathrm{GeV}$. In Fig. 2(a) we show $\operatorname{BR}\left(\tilde{t}_{1} \rightarrow b W^{+} \tilde{\chi}_{1}^{0}\right), \operatorname{BR}\left(\tilde{t}_{1} \rightarrow c \tilde{\chi}_{1}^{0}\right), \operatorname{BR}\left(\tilde{t}_{1} \rightarrow b e^{+} \tilde{\nu}_{e}\right)+\operatorname{BR}\left(\tilde{t}_{1} \rightarrow b \nu_{e} \tilde{e}_{L}^{+}\right)$, and $\operatorname{BR}\left(\tilde{t}_{1} \rightarrow b \tau^{+} \tilde{\nu}_{\tau}\right)+\operatorname{BR}\left(\tilde{t}_{1} \rightarrow b \nu_{\tau} \tilde{\tau}_{1}^{+}\right)+\operatorname{BR}\left(\tilde{t}_{1} \rightarrow b \nu_{\tau} \tilde{\tau}_{2}^{+}\right)$. Here we have not included the possibility of the decay $\tilde{t}_{1} \rightarrow b H^{+} \tilde{\chi}_{1}^{0}$ because with this parameter set there exists no value of $m_{A^{0}}$ which simultaneously allows this decay and fulfills the condition $m_{h^{0}} \geq 71 \mathrm{GeV}$ [12] (we have used the MSSM formula for the calculation of $m_{H^{+}}$including 1-loop corrections as given in [13]). However, we will discuss this decay later on. We have summed up those branching ratios for the decays into sleptons that give the same final state, for example:

$$
\tilde{t}_{1} \rightarrow b \nu_{\tau} \tilde{\tau}_{1}^{+} \rightarrow b \tau^{+} \nu_{\tau} \tilde{\chi}_{1}^{0}, \quad \tilde{t}_{1} \rightarrow b \tau^{+} \tilde{\nu}_{\tau} \rightarrow b \tau^{+} \nu_{\tau} \tilde{\chi}_{1}^{0}
$$

Note, that in the above cases the assumption $m_{\tilde{t}_{1}}-m_{b}<m_{\tilde{\chi}_{1}^{+}}$implies $m_{\tilde{\chi}_{1}^{+}}>m_{\tilde{l}}$. Therefore, the sleptons can only decay into the corresponding lepton plus $\tilde{\chi}_{1}^{0}$ except for a small parameter region where the decay into $\tilde{\chi}_{2}^{0}$ is possible. However, this decay is negligible due to kinematics in that region. The branching ratios for decays into $\tilde{\mu}_{L}$ or $\tilde{\nu}_{\mu}$ are practically the same as those into $\tilde{e}_{L}$ or $\tilde{\nu}_{e}$. For this set of parameters $\operatorname{BR}\left(\tilde{t}_{1} \rightarrow c \tilde{\chi}_{1}^{0}\right)$ is $O\left(10^{-4}\right)$ independent of $\cos \theta_{\tilde{t}}$ and therefore negligible. Near $\cos \theta_{\tilde{t}}=-0.3 \mathrm{BR}\left(\tilde{t}_{1} \rightarrow b W^{+} \tilde{\chi}_{1}^{0}\right)$ is almost $100 \%$ because the $\tilde{t}_{1-} \tilde{\chi}_{1}^{+}-b$ coupling $l_{11}^{\tilde{t}}$ vanishes. We have found that the decay $\tilde{t}_{1} \rightarrow b W^{+} \tilde{\chi}_{1}^{0}$ is dominated by the $t$-quark exchange. In many cases the interference term between $t$ and $\tilde{\chi}_{1,2}^{+}$ is more important than the $\tilde{\chi}_{1,2}^{+}$exchange. Moreover, we have found that the contribution from sbottom exchange is in general negligible.

In Fig. 2(b) the branching ratios for the various decays into sleptons are shown. For small $\tan \beta$ sleptons couple mainly to the gaugino components of $\tilde{\chi}_{1}^{+}$. This leads to $\operatorname{BR}\left(\tilde{t}_{1} \rightarrow\right.$ $\left.b \nu_{e} \tilde{e}_{L}^{+}\right)>\operatorname{BR}\left(\tilde{t}_{1} \rightarrow b \nu_{\tau} \tilde{\tau}_{1,2}^{+}\right)$because $\cos \theta_{\tilde{\tau}} \simeq 0.68$. The decays into sneutrinos are preferred by kinematics while the decay into $\tilde{\tau}_{2}$ is suppressed by the same reason (Table $\mathbb{Q}$ ). Moreover, the matrix elements Eqs. (17) and (18) for the decays into charged and neutral sleptons have a different structure in the limit $m_{b}, m_{l} \rightarrow 0$ :

$$
\begin{aligned}
M_{\tilde{t}_{1} \rightarrow b l^{+} \tilde{\nu}_{l}} & \sim m_{\tilde{\chi}_{i}} \bar{u}\left(p_{b}\right) P_{R} v\left(p_{l}\right), \\
M_{\tilde{t}_{1} \rightarrow b \nu_{l} \tilde{i}_{k}^{+}} & \sim \bar{u}\left(p_{b}\right) P_{R} \not p_{\tilde{\chi}_{i}^{+}} v\left(p_{\nu_{l}}\right) .
\end{aligned}
$$

This leads to different decay widths even in the limit of equal slepton masses.

In Figs. 3 (a) and (b) we show the branching ratios as a function of $\tan \beta$ for $\cos \theta_{\tilde{t}}=0.6$ and the other parameters as above. For small $\tan \beta$ the decay into $\tilde{t}_{1} \rightarrow b W^{+} \tilde{\chi}_{1}^{0}$ is the most important one. The branching ratios for the decays into sleptons decrease with increasing $\tan \beta$ except for the decay into $\tilde{\tau}_{1}$. This results from: (i) for increasing $\tan \beta$ the gaugino component of $\tilde{\chi}_{1}^{+}$decreases while its mass increases, (ii) the masses of the sleptons increase with increasing $\tan \beta$, except $m_{\tilde{\tau}_{1}}$ which decreases, and (iii) the $\tau$ Yukawa coupling increases. These facts lead to the dominance of $\tilde{t}_{1} \rightarrow b \nu_{\tau} \tilde{\tau}_{1}$ for large $\tan \beta$ as can be seen in Fig. (3)(b). In addition, the decay into $c \tilde{\chi}_{1}^{0}$ gains some importance for large $\tan \beta$ because its width is proportional to the bottom Yukawa coupling in the approximation of [1]].

The assumption that no two-body decays be allowed at tree level implies that $m_{\tilde{\chi}_{1}^{+}}>$ $m_{\tilde{t}_{1}}-m_{b}$. Therefore, one expects an increase of $\operatorname{BR}\left(\tilde{t}_{1} \rightarrow b W^{+} \tilde{\chi}_{1}^{0}\right)$ if $m_{\tilde{t}_{1}}$ increases, because 
the decay into $b W^{+} \tilde{\chi}_{1}^{0}$ is dominated by the $t$ exchange whereas for the decays into sleptons $\tilde{\chi}_{1}^{+}$exchange dominates. This is demonstrated in Figs. 1(a) and (b) where we have fixed $m_{\tilde{t}_{1}}=350 \mathrm{GeV}$. Here also the decay into $b H^{+} \tilde{\chi}_{1}^{0}$ is possible. However, this channel is in general suppressed by kinematics. We have not found any case with $m_{H^{+}} \leq 120 \mathrm{GeV}$ while $m_{h^{0}}>75 \mathrm{GeV}$ [12.

These general features still hold if $\tan \beta$ increases as can be seen in Figs. 5(a) and (b). Here we have fixed $\cos \theta_{\tilde{t}}=0.7$. In accordance with the discussion above, the decay into $\tilde{t}_{1} \rightarrow$ $b \nu_{\tau} \tilde{\tau}_{1}$ gains importance with increasing $\tan \beta$. Note, that for large $\tan \beta \operatorname{BR}\left(\tilde{t}_{1} \rightarrow b H^{+} \tilde{\chi}_{1}^{0}\right)$ decreases since $m_{H^{+}}$increases due to radiative corrections. However, there are scenarios where the decay $\tilde{t}_{1} \rightarrow b H^{+} \tilde{\chi}_{1}^{0}$ becomes important. This can be seen in Fig. 6 where the branching ratios are shown as a function of $M_{\tilde{D}}$ for $m_{A^{0}}=90 \mathrm{GeV}, \tan \beta=30$, and the other parameters as in Tab. 四. At the lower end of the $M_{\tilde{D}}$ range we get $m_{H^{+}}=114 \mathrm{GeV}$. Moreover, $m_{\tilde{b}_{1}}$ is approximately $m_{\tilde{t}_{1}}-m_{W}$ leading to an enhancement of this width. We have found that contrary to the case $\tilde{t}_{1} \rightarrow b W^{+} \tilde{\chi}_{1}^{0}$ for the decays $\tilde{t}_{1} \rightarrow b H^{+} \tilde{\chi}_{1}^{0}$ sbottom exchange can be important. This is a consequence of the different spin structure of the corresponding matrix elements (Eqs. (14) and (15)) and because of the large bottom Yukawa coupling. The decrease in $\operatorname{BR}\left(\tilde{t}_{1} \rightarrow b H^{+} \tilde{\chi}_{1}^{0}\right)$ for $M_{\tilde{D}} \geq 450 \mathrm{GeV}$ is mainly due to the fact that $m_{H^{+}}$ grows with increasing $M_{\tilde{D}}$.

Finally, we want to discuss a scenario which is within the reach of an upgraded Tevatron. Here we refer to the examples of [11]. In general the decays into sleptons clearly dominate when they are kinematically allowed (except the case when the couplings of the top squark to the lighter chargino nearly vanishes). In the case $m_{\tilde{t}_{1}}=170 \mathrm{GeV}, \cos \theta_{\tilde{t}}=-0.7, M_{\tilde{D}}=$ $M_{\tilde{Q}}=500 \mathrm{GeV}, A_{b}=A_{\tau}=-350 \mathrm{GeV}, \mu=-1000 \mathrm{GeV}, M=165 \mathrm{GeV}$ and $\tan \beta=2$ (scenario b of Table I in [11]) we obtain: $\operatorname{BR}\left(\tilde{t}_{1} \rightarrow b \nu_{e} \tilde{e}_{L}^{+}\right)=2.8 \%, \operatorname{BR}\left(\tilde{t}_{1} \rightarrow b \nu_{\tau} \tilde{\tau}_{1}\right)=10.5 \%$, $\operatorname{BR}\left(\tilde{t}_{1} \rightarrow b \nu_{\tau} \tilde{\tau}_{2}\right)=2 \times 10^{-3} \%, \operatorname{BR}\left(\tilde{t}_{1} \rightarrow b e^{+} \tilde{\nu}_{e}\right)=28.1 \%$, and $\operatorname{BR}\left(\tilde{t}_{1} \rightarrow b \tau^{+} \tilde{\nu}_{\tau}\right)=27.8 \%$. The order of magnitude is independent of $\mu$ and $\cos \theta_{\tilde{t}}$ because the lighter chargino is mainly gaugino-like in the parameter space where the decay $\tilde{t}_{1} \rightarrow b W^{+} \tilde{\chi}_{1}^{0}$ is possible (see Fig. $2 \mathrm{~b}$ of [11]). For increasing $\tan \beta$ we have found a similar behaviour as in Figs. 3 and 5: dominance of the decay $\tilde{t}_{1} \rightarrow b \nu_{\tau} \tilde{\tau}_{1}^{+}$, an increase of $\tilde{t}_{1} \rightarrow c \tilde{\chi}_{1}^{0}$ and a decrease of all other decay channels.

\section{CONCLUSIONS}

We have calculated the three-body decays $\tilde{t}_{1} \rightarrow b H^{+} \tilde{\chi}_{1}^{0}, \tilde{t}_{1} \rightarrow b \nu_{l} \tilde{l}_{i}^{+}$, and $\tilde{t}_{1} \rightarrow b l^{+} \tilde{\nu}_{l}$ $(l=e, \mu, \tau)$ including all terms proportional to $m_{b}, m_{\tau}$, and all Yukawa couplings. We have compared these decays with $\tilde{t}_{1} \rightarrow c \tilde{\chi}_{1}^{0}[10]$ and $\tilde{t}_{1} \rightarrow b W^{+} \tilde{\chi}_{1}^{0}$ [11]. These decays are competitive in that part of the parameter space - accessible at an upgraded Tevatron, the LHC or a future lepton collider - where the tree-level two-body decays $\tilde{t}_{1} \rightarrow b \tilde{\chi}_{i}^{+}$and $\tilde{t}_{1} \rightarrow t \tilde{\chi}_{j}^{0}$ are kinematically forbidden. We have found that for $m_{\tilde{t}_{1}} \leq 200 \mathrm{GeV}$ the decays into sleptons dominate due to kinematics. In the range $200 \mathrm{GeV} \leq m_{\tilde{t}_{1}} \leq 300 \mathrm{GeV}$ all the decays mentioned above compete with each other. The branching ratios depend crucially

on the coupling of $\tilde{t}_{1}$ to $\tilde{\chi}_{1}^{+}$, implying that one can get information on the mixing angle of the top squarks, once the chargino properties are known. For heavier top squark masses the decay into $b W^{+} \tilde{\chi}_{1}^{0}$, mainly proceeding via a virtual $t$ quark, is in general the most important one. 
In addition, we have found that for small $\tan \beta$ the decays into sneutrinos are more important than the decays into charged sleptons. This is a result of the different spin structures of the corresponding matrix elements. For large $\tan \beta$ the decay into the lighter stau becomes important due to the large tau Yukawa coupling (implying also a smaller stau mass).

The decay into $b H^{+} \tilde{\chi}_{1}^{0}$ is kinematically suppressed because the existing mass bounds on the neutral Higgs bosons also imply a lower bound on $m_{H^{+}}$. However, in scenarios where radiative corrections decrease $m_{H^{+}}$and where at the same time $m_{\tilde{t}_{1}}>m_{\tilde{b}_{1}}$ we have found branching ratios of the order of $30 \%$.

The large variety of possible three-body decay modes implies the chance to determine

the properties of $\tilde{t}_{1}$ also when higher order decays are dominant. Clearly, a detailed Monte Carlo study will be necessary to see how the different channels can be separated.

\section{ACKNOWLEDGMENTS}

I thank A. Bartl, H. Eberl, T. Gajdosik, S. Kraml, and W. Majerotto for many helpful discussions and the inspiring atmosphere. I am grateful to J.W.F. Valle for the kind hospitality and the pleasant atmosphere at the Departamento de Física Teórico. This work was supported by the "Fonds zur Förderung der wissenschaftlichen Forschung" of Austria, project No. P10843-PHY, and by the EEC under the TMR contract ERBFMRX-CT960090.

\section{APPENDIX A: FORMULAS FOR THE THREE-BODY DECAY WIDTHS}

In the subsequent sections the formulas for the decay widths are listed which have been omitted in Sec. II.

\section{The width $\Gamma\left(\tilde{t}_{1} \rightarrow H^{+} b \tilde{\chi}_{1}^{0}\right)$}

The decay width is given by

$$
\begin{aligned}
\Gamma\left(\tilde{t}_{1}\right. & \left.\rightarrow H^{+} b \tilde{\chi}_{1}^{0}\right)= \\
= & \frac{\alpha^{2}}{16 \pi m_{\tilde{t}_{1}}^{3} \sin ^{4} \theta_{W}} \int_{\left(m_{b}+m_{\tilde{\chi}_{1}^{0}}\right)^{2}}^{\left(m_{\tilde{t}_{1}}-m_{H^{+}}\right)^{2}} d s\left(G_{\tilde{\chi}^{+} \tilde{\chi}^{+}}+G_{\tilde{\chi}^{+} t}+G_{\tilde{\chi}^{+} \tilde{b}}+G_{t t}+G_{t \tilde{b}}+G_{\tilde{b} \tilde{b}}\right)
\end{aligned}
$$

with

$$
\begin{aligned}
G_{\tilde{\chi}^{+} \tilde{\chi}^{+}}=\sum_{i=1}^{2} & {\left[\left(a_{i 1}+a_{i 2} s\right) J_{t}^{0}\left(m_{\tilde{t}_{1}}^{2}+m_{H^{+}}^{2}+m_{b}^{2}+m_{\tilde{\chi}_{1}^{0}}^{2}-m_{\tilde{\chi}_{i}^{+}}^{2}-s, \Gamma_{\tilde{\chi}_{i}^{+}} m_{\tilde{\chi}_{i}^{+}}\right)\right.} \\
+ & \left(a_{i 3}+a_{i 4} s\right) J_{t}^{1}\left(m_{\tilde{t}_{1}}^{2}+m_{H^{+}}^{2}+m_{b}^{2}+m_{\tilde{\chi}_{1}^{0}}^{2}-m_{\tilde{\chi}_{i}^{+}}^{2}-s, \Gamma_{\tilde{\chi}_{i}^{+}} m_{\tilde{\chi}_{i}^{+}}\right) \\
& \left.+a_{i 4} J_{t}^{2}\left(m_{\tilde{t}_{1}}^{2}+m_{H^{+}}^{2}+m_{b}^{2}+m_{\tilde{\chi}_{1}^{0}}^{2}-m_{\tilde{\chi}_{i}^{+}}^{2}-s, \Gamma_{\tilde{\chi}_{i}^{+}} m_{\tilde{\chi}_{i}^{+}}\right)\right]
\end{aligned}
$$




$$
\begin{aligned}
& +\left(a_{31}+a_{32} s\right) J_{t t}^{0}\left(m_{\tilde{t}_{1}}^{2}+m_{H^{+}}^{2}+m_{b}^{2}+m_{\tilde{\chi}_{1}^{0}}^{2}-m_{\tilde{\chi}_{1}^{+}}^{2}-s, \Gamma_{\tilde{\chi}_{1}^{+}} m_{\tilde{\chi}_{1}^{+}}\right. \\
& \left., m_{\tilde{t}_{1}}^{2}+m_{H^{+}}^{2}+m_{b}^{2}+m_{\tilde{\chi}_{1}^{0}}^{2}-m_{\tilde{\chi}_{2}^{+}}^{2}-s, \Gamma_{\tilde{\chi}_{2}^{+}} m_{\tilde{\chi}_{2}^{+}}\right) \\
& +\left(a_{33}+a_{34} s\right) J_{t t}^{1}\left(m_{\tilde{t}_{1}}^{2}+m_{H^{+}}^{2}+m_{b}^{2}+m_{\tilde{\chi}_{1}^{0}}^{2}-m_{\tilde{\chi}_{1}^{+}}^{2}-s, \Gamma_{\tilde{\chi}_{1}^{+}} m_{\tilde{\chi}_{1}^{+}}\right. \\
& \left., m_{\tilde{t}_{1}}^{2}+m_{H^{+}}^{2}+m_{b}^{2}+m_{\tilde{\chi}_{1}^{0}}^{2}-m_{\tilde{\chi}_{2}^{+}}^{2}-s, \Gamma_{\tilde{\chi}_{2}^{+}} m_{\tilde{\chi}_{2}^{+}}\right) \\
& +a_{34} J_{t t}^{2}\left(m_{\tilde{t}_{1}}^{2}+m_{H^{+}}^{2}+m_{b}^{2}+m_{\tilde{\chi}_{1}^{0}}^{2}-m_{\tilde{\chi}_{1}^{+}}^{2}-s, \Gamma_{\tilde{\chi}_{1}^{+}} m_{\tilde{\chi}_{1}^{+}}\right. \\
& \left., m_{\tilde{t}_{1}}^{2}+m_{H^{+}}^{2}+m_{b}^{2}+m_{\tilde{\chi}_{1}^{0}}^{2}-m_{\tilde{\chi}_{2}^{+}}^{2}-s, \Gamma_{\tilde{\chi}_{2}^{+}} m_{\tilde{\chi}_{2}^{+}}\right), \\
& G_{\tilde{\chi}^{+} t}=\sum_{i=1}^{2}\left[\left(b_{i 1}+b_{i 2} s\right) J_{t t}^{0}\left(m_{\tilde{t}_{1}}^{2}+m_{H^{+}}^{2}+m_{b}^{2}+m_{\tilde{\chi}_{1}^{0}}^{2}-m_{\tilde{\chi}_{1}^{+}}^{2}-s,-\Gamma_{\tilde{\chi}_{1}^{+}} m_{\tilde{\chi}_{1}^{+}}, m_{t}^{2}, \Gamma_{t} m_{t}\right)\right. \\
& +\left(b_{i 3}+b_{i 4} s\right) J_{t t}^{1}\left(m_{\tilde{t}_{1}}^{2}+m_{H^{+}}^{2}+m_{b}^{2}+m_{\tilde{\chi}_{1}^{0}}^{2}-m_{\tilde{\chi}_{1}^{+}}^{2}-s,-\Gamma_{\tilde{\chi}_{1}^{+}} m_{\tilde{\chi}_{1}^{+}}, m_{t}^{2}, \Gamma_{t} m_{t}\right) \\
& \left.+b_{i 4} J_{t t}^{2}\left(m_{\tilde{t}_{1}}^{2}+m_{H^{+}}^{2}+m_{b}^{2}+m_{\tilde{\chi}_{1}^{0}}^{2}-m_{\tilde{\chi}_{1}^{+}}^{2}-s,-\Gamma_{\tilde{\chi}_{1}^{+}} m_{\tilde{\chi}_{1}^{+}}, m_{t}^{2}, \Gamma_{t} m_{t}\right)\right] \text {, } \\
& G_{\tilde{\chi}+\tilde{b}}=\sum_{k, i=1}^{2}\left[\left(c_{i k 1}+c_{i k 2} s\right)\right. \\
& * J_{s t}^{0}\left(m_{\tilde{b}_{k}}^{2}, \Gamma_{\tilde{b}_{k}} m_{\tilde{b}_{k}}, m_{\tilde{t}_{1}}^{2}+m_{H^{+}}^{2}+m_{b}^{2}+m_{\tilde{\chi}_{1}^{0}}^{2}-m_{\tilde{\chi}_{1}^{+}}^{2}-s,-\Gamma_{\tilde{\chi}_{1}^{+}} m_{\tilde{\chi}_{1}^{+}}\right) \\
& \left.+c_{i k 3} J_{s t}^{1}\left(m_{\tilde{b}_{k}}^{2}, \Gamma_{\tilde{b}_{k}} m_{\tilde{b}_{k}}, m_{\tilde{t}_{1}}^{2}+m_{H^{+}}^{2}+m_{b}^{2}+m_{\tilde{\chi}_{1}^{0}}^{2}-m_{\tilde{\chi}_{1}^{+}}^{2}-s,-\Gamma_{\tilde{\chi}_{1}^{+}} m_{\tilde{\chi}_{1}^{+}}\right)\right] \text {, } \\
& G_{t t}=\left(d_{1}+d_{2} s\right) J_{t}^{0}\left(m_{t}^{2}, \Gamma_{t} m_{t}\right)+\left(d_{3}+d_{4} s\right) J_{t}^{1}\left(m_{t}^{2}, \Gamma_{t} m_{t}\right)+d_{4} J_{t}^{2}\left(m_{t}^{2}, \Gamma_{t} m_{t}\right) \text {, } \\
& G_{t \tilde{b}}=\sum_{k=1}^{2}\left[\left(e_{k 1}+e_{k 2} s\right) J_{s t}^{0}\left(m_{\tilde{b}_{k}}^{2}, \Gamma_{\tilde{b}_{k}} m_{\tilde{b}_{k}}, m_{t}^{2}, \Gamma_{t} m_{t}\right)+e_{k 3} J_{s t}^{1}\left(m_{\tilde{b}_{k}}^{2}, \Gamma_{\tilde{b}_{k}} m_{\tilde{b}_{k}}, m_{t}^{2}, \Gamma_{t} m_{t}\right)\right] \text {, } \\
& G_{\tilde{b} \tilde{b}}=\frac{\sqrt{\lambda\left(s, m_{\tilde{t}_{1}}^{2}, m_{H^{+}}^{2}\right) \lambda\left(s, m_{\tilde{\chi}_{1}^{0}}^{2}, m_{b}^{2}\right)}}{s} \\
& *\left\{\sum_{k=1}^{2} \frac{\left(f_{k 1}+f_{k 2} s\right)}{\left(s-m_{\tilde{b}_{k}}^{2}\right)^{2}+\Gamma_{\tilde{b}_{k}}^{2} m_{\tilde{b}_{k}}^{2}}+\operatorname{Re}\left[\frac{\left(f_{31}+f_{32} s\right)}{\left(s-m_{\tilde{b}_{1}}^{2}+i \Gamma_{\tilde{b}_{1}} m_{\tilde{b}_{1}}\right)\left(s-m_{\tilde{b}_{2}}^{2}-i \Gamma_{\tilde{b}_{2}} m_{\tilde{b}_{2}}\right)}\right]\right\} .
\end{aligned}
$$

The integrals $J_{t, t t, s t}^{0,1,2}$ are:

$$
\begin{aligned}
& J_{t}^{i}\left(m_{1}^{2}, m_{1} \Gamma_{1}\right)=\int_{t_{\text {min }}}^{t_{\text {max }}} d t \frac{t^{i}}{\left(t-m_{1}^{2}\right)^{2}+m_{1}^{2} \Gamma_{1}^{2}}, \\
& J_{t t}^{i}\left(m_{1}^{2}, m_{1} \Gamma_{1}, m_{2}^{2}, m_{2} \Gamma_{2}\right)=\operatorname{Re} \int_{t_{\text {min }}}^{t_{\text {max }}} d t \frac{t^{i}}{\left(t-m_{1}^{2}+i m_{1} \Gamma_{1}\right)\left(t-m_{2}^{2}-i m_{2} \Gamma_{2}\right)}, \\
& J_{s t}^{i}\left(m_{1}^{2}, m_{1} \Gamma_{1}, m_{2}^{2}, m_{2} \Gamma_{2}\right)=\operatorname{Re} \frac{1}{s-m_{1}^{2}+i m_{1} \Gamma_{1}} \int_{t_{\min }}^{t_{\text {max }}} d t \frac{t^{i}}{\left(t-m_{2}^{2}-i m_{2} \Gamma_{2}\right)}
\end{aligned}
$$


with $i=0,1,2$. Their integration range is given by

$$
\begin{aligned}
t_{\min }^{\max }= & \frac{m_{\tilde{t}_{1}}^{2}+m_{b}^{2}+m_{H^{+}}^{2}+m_{\tilde{\chi}_{1}^{0}}^{2}-s}{2}-\frac{\left(m_{\tilde{t}_{1}}^{2}-m_{H^{+}}^{2}\right)\left(m_{\tilde{\chi}_{1}^{0}}^{2}-m_{b}^{2}\right)}{2 s} \\
& \pm \frac{\sqrt{\lambda\left(s, m_{\tilde{t}_{1}}^{2}, m_{H^{+}}^{2}\right) \lambda\left(s, m_{\tilde{\chi}_{1}^{0}}^{2}, m_{b}^{2}\right)}}{2 s},
\end{aligned}
$$

where $s=\left(p_{\tilde{t}_{1}}-p_{H^{+}}\right)^{2}$ and $t=\left(p_{\tilde{t}_{1}}-p_{t}\right)^{2}$ are the usual Mandelstam variables. Note, that $-\Gamma_{\tilde{\chi}_{1}^{+}} m_{\tilde{\chi}_{1}^{+}}$appears in the entries of the integrals $G_{\tilde{\chi}^{+} \tilde{b}_{j}}$ and $G_{\tilde{\chi}^{+} t}$ because the chargino is exchanged in the $u$-channel in our convention. The coefficients are given by:

$$
\begin{aligned}
& a_{11}=-4 k_{11}^{\tilde{t}} l_{11}^{\tilde{t}} Q_{11}^{L \prime} Q_{11}^{R \prime} m_{b} m_{\tilde{\chi}_{1}^{0}}\left(m_{b}^{2}+m_{\tilde{\chi}_{1}^{+}}^{2}+m_{\tilde{\chi}_{1}^{0}}^{2}+m_{\tilde{t}_{1}}^{2}+m_{H^{+}}^{2}\right) \\
& -2 Q_{11}^{L \prime} Q_{11}^{R \prime}\left(\left(k_{11}^{\tilde{t}}\right)^{2}+\left(l_{11}^{\tilde{t}}\right)^{2}\right) m_{\tilde{\chi}_{1}^{0}} m_{\tilde{\chi}_{1}^{+}}\left(2 m_{b}^{2}+m_{\tilde{\chi}_{1}^{0}}^{2}+m_{H^{+}}^{2}\right) \\
& -2 k_{11}^{\tilde{t}} l_{11}^{\tilde{t}}\left(\left(Q_{11}^{L \prime}\right)^{2}+\left(Q_{11}^{R \prime}\right)^{2}\right) m_{b} m_{\tilde{\chi}_{1}^{+}}\left(m_{b}^{2}+2 m_{\tilde{\chi}_{1}^{0}}^{2}+m_{\tilde{t}_{1}}^{2}\right) \\
& -\left(\left(k_{11}^{\tilde{t}}\right)^{2}\left(Q_{11}^{R \prime}\right)^{2}+\left(l_{11}^{\tilde{t}}\right)^{2}\left(Q_{11}^{L \prime}\right)^{2}\right) \\
& *\left[\left(m_{b}^{2}+m_{\tilde{\chi}_{1}^{0}}^{2}\right)^{2}+\left(m_{b}^{2}+m_{H^{+}}^{2}\right)\left(m_{\tilde{\chi}_{1}^{0}}^{2}+m_{\tilde{t}_{1}}^{2}\right)\right] \\
& -\left(\left(k_{11}^{\tilde{t}}\right)^{2}\left(Q_{11}^{L \prime}\right)^{2}+\left(l_{11}^{\tilde{t}}\right)^{2}\left(Q_{11}^{R \prime}\right)^{2}\right) m_{\tilde{\chi}_{1}^{+}}^{2}\left(m_{b}^{2}+m_{\tilde{\chi}_{1}^{0}}^{2}\right), \\
& a_{12}=4 k_{11}^{\tilde{t}} l_{11}^{\tilde{t}} Q_{11}^{L \prime} Q_{11}^{R \prime} m_{b} m_{\tilde{\chi}_{1}^{0}}+\left(\left(k_{11}^{\tilde{t}}\right)^{2}\left(Q_{11}^{R \prime}\right)^{2}+\left(l_{11}^{\tilde{t}}\right)^{2}\left(Q_{11}^{L \prime}\right)^{2}\right)\left(m_{b}^{2}+m_{\tilde{\chi}_{1}^{0}}^{2}\right) \\
& +2 k_{11}^{\tilde{t}} l_{11}^{\tilde{t}}\left(\left(Q_{11}^{L \prime}\right)^{2}+\left(Q_{11}^{R \prime}\right)^{2}\right) m_{b} m_{\tilde{\chi}_{1}^{+}}+2 Q_{11}^{L \prime} Q_{11}^{R \prime}\left(\left(k_{11}^{\tilde{t}}\right)^{2}+\left(l_{11}^{\tilde{t}}\right)^{2}\right) m_{\tilde{\chi}_{1}^{0}} m_{\tilde{\chi}_{1}^{+}} \\
& +\left(\left(k_{11}^{\tilde{t}}\right)^{2}\left(Q_{11}^{L \prime}\right)^{2}+\left(l_{11}^{\tilde{t}}\right)^{2}\left(Q_{11}^{R \prime}\right)^{2}\right) m_{\tilde{\chi}_{1}^{+}}^{2}, \\
& a_{13}=4 k_{11}^{\tilde{t}} l_{11}^{\tilde{t}} Q_{11}^{L \prime} Q_{11}^{R \prime} m_{b} m_{\tilde{\chi}_{1}^{0}}+2 Q_{11}^{L \prime} Q_{11}^{R \prime}\left(\left(k_{11}^{\tilde{t}}\right)^{2}+\left(l_{11}^{\tilde{t}}\right)^{2}\right) m_{\tilde{\chi}_{1}^{0}} m_{\tilde{\chi}_{1}^{+}} \\
& +\left(\left(k_{11}^{\tilde{t}}\right)^{2}\left(Q_{11}^{R \prime}\right)^{2}+\left(l_{11}^{\tilde{t}}\right)^{2}\left(Q_{11}^{L \prime}\right)^{2}\right)\left(2 m_{b}^{2}+2 m_{\tilde{\chi}_{1}^{0}}^{2}+m_{H^{+}}^{2}+m_{\tilde{t}_{1}}^{2}\right) \\
& +2 k_{11}^{\tilde{t}} l_{11}^{\tilde{t}}\left(\left(Q_{11}^{L \prime}\right)^{2}+\left(Q_{11}^{R \prime}\right)^{2}\right) m_{b} m_{\tilde{\chi}_{1}^{+}} \text {, } \\
& a_{14}=-\left(k_{11}^{\tilde{t}}\right)^{2}\left(Q_{11}^{R \prime}\right)^{2}-\left(l_{11}^{\tilde{t}}\right)^{2}\left(Q_{11}^{L \prime}\right)^{2} \text {, } \\
& a_{31}=-2\left(l_{11}^{\tilde{t}} l_{12}^{\tilde{t}} Q_{12}^{L \prime} Q_{11}^{R \prime}+k_{11}^{\tilde{t}} k_{12}^{\tilde{t}} Q_{11}^{L \prime} Q_{12}^{R}{ }^{\prime}\right) m_{\tilde{\chi}_{1}^{0}} m_{\tilde{\chi}_{1}^{+}}\left(m_{\tilde{\chi}_{1}^{0}}^{2}+m_{H^{+}}^{2}+2 m_{b}^{2}\right) \\
& -4\left(k_{11}^{\tilde{t}} l_{12}^{\tilde{t}} Q_{12}^{L \prime} Q_{11}^{R \prime}+k_{12}^{\tilde{t}} l_{11}^{\tilde{t}} Q_{11}^{L \prime} Q_{12}^{R \prime}\right) m_{b} m_{\tilde{\chi}_{1}^{0}}\left(m_{\tilde{\chi}_{1}^{0}}^{2}+m_{H^{+}}^{2}+m_{b}^{2}+m_{\tilde{t}_{1}}^{2}\right) \\
& -4\left(k_{12}^{\tilde{t}} l_{11}^{\tilde{t}} Q_{12}^{L \prime} Q_{11}^{R \prime}+k_{11}^{\tilde{t}} l_{12}^{\tilde{t}} Q_{11}^{L \prime} Q_{12}^{R \prime}\right) m_{b} m_{\tilde{\chi}_{1}^{0}} m_{\tilde{\chi}_{1}^{+}} m_{\tilde{\chi}_{2}^{+}} \\
& -2\left(k_{11}^{\tilde{t}} k_{12}^{\tilde{t}} Q_{12}^{L \prime} Q_{11}^{R \prime}+l_{11}^{\tilde{t}} l_{12}^{\tilde{t}} Q_{11}^{L \prime} Q_{12}^{R \prime}\right) m_{\tilde{\chi}_{1}^{0}} m_{\tilde{\chi}_{2}^{+}}\left(m_{\tilde{\chi}_{1}^{0}}^{2}+m_{H^{+}}^{2}+2 m_{b}^{2}\right) \\
& -2\left(l_{11}^{\tilde{t}} l_{12}^{\tilde{t}} Q_{11}^{L \prime} Q_{12}^{L \prime}+k_{11}^{\tilde{t}} k_{12}^{\tilde{t}} Q_{11}^{R \prime} Q_{12}^{R \prime}\right) \\
& *\left[\left(m_{b}^{2}+m_{\tilde{\chi}_{1}^{0}}^{2}\right)^{2}+\left(m_{\tilde{\chi}_{1}^{0}}^{2}+m_{\tilde{t}_{1}}^{2}\right)\left(m_{b}^{2}+m_{H^{+}}^{2}\right)\right]
\end{aligned}
$$




$$
\begin{aligned}
& -2\left(k_{11}^{\tilde{t}} l_{12}^{\tilde{t}} Q_{11}^{L \prime} Q_{12}^{L \prime}+k_{12}^{\tilde{t}} l_{11}^{\tilde{t}} Q_{11}^{R \prime} Q_{12}^{R \prime}\right) m_{b} m_{\tilde{\chi}_{1}^{+}}\left(m_{b}^{2}+m_{\tilde{t}_{1}}^{2}+2 m_{\tilde{\chi}_{1}^{0}}^{2}\right) \\
& -2\left(k_{12}^{\tilde{t}} l_{11}^{\tilde{t}} Q_{11}^{L \prime} Q_{12}^{L \prime}+k_{11}^{\tilde{t}} l_{12}^{\tilde{t}} Q_{11}^{R \prime} Q_{12}^{R \prime}\right) m_{b} m_{\tilde{\chi}_{2}^{+}}\left(m_{b}^{2}+m_{\tilde{t}_{1}}^{2}+2 m_{\tilde{\chi}_{1}^{0}}^{2}\right) \\
& -2\left(k_{11}^{\tilde{t}} k_{12}^{\tilde{t}} Q_{11}^{L \prime} Q_{12}^{L \prime}+l_{11}^{\tilde{t}} l_{12}^{\tilde{t}} Q_{11}^{R \prime} Q_{12}^{R \prime}\right) m_{\tilde{\chi}_{1}^{+}} m_{\tilde{\chi}_{2}^{+}}\left(m_{\tilde{\chi}_{1}^{0}}^{2}+m_{b}^{2}\right) \text {, } \\
& a_{32}=2\left(l_{11}^{\tilde{t}} l_{12}^{\tilde{t}} Q_{12}^{L \prime} Q_{11}^{R \prime}+k_{11}^{\tilde{t}} k_{12}^{\tilde{t}} Q_{11}^{L \prime} Q_{12}^{R \prime}\right) m_{\tilde{\chi}_{1}^{0}} m_{\tilde{\chi}_{1}^{+}} \\
& +4\left(k_{11}^{\tilde{t}} l_{12}^{\tilde{t}} Q_{12}^{L \prime} Q_{11}^{R \prime}+k_{12}^{\tilde{t}} l_{11}^{\tilde{t}} Q_{11}^{L \prime} Q_{12}^{R \prime}\right) m_{b} m_{\tilde{\chi}_{1}^{0}} \\
& +2\left(k_{11}^{\tilde{t}} k_{12}^{\tilde{t}} Q_{12}^{L \prime} Q_{11}^{R \prime}+l_{11}^{\tilde{t}} l_{12}^{\tilde{t}} Q_{11}^{L \prime} Q_{12}^{R \prime}\right) m_{\tilde{\chi}_{1}^{0}} m_{\tilde{\chi}_{2}^{+}} \\
& +2\left(l_{11}^{\tilde{t}} l_{12}^{\tilde{t}} Q_{11}^{L \prime} Q_{12}^{L \prime}+k_{11}^{\tilde{t}} k_{12}^{\tilde{t}} Q_{11}^{R \prime} Q_{12}^{R \prime}\right)\left(m_{b}^{2}+m_{\tilde{\chi}_{1}^{0}}^{2}\right) \\
& +2\left(k_{11}^{\tilde{t}} l_{12}^{\tilde{t}} Q_{11}^{L \prime} Q_{12}^{L \prime}+k_{12}^{\tilde{t}} l_{11}^{\tilde{t}} Q_{11}^{R \prime} Q_{12}^{R \prime}\right) m_{b} m_{\tilde{\chi}_{1}^{+}} \\
& +2\left(k_{12}^{\tilde{t}} l_{11}^{\tilde{t}} Q_{11}^{L \prime} Q_{12}^{L \prime}+k_{11}^{\tilde{t}} l_{12}^{\tilde{t}} Q_{11}^{R \prime} Q_{12}^{R \prime}\right) m_{b} m_{\tilde{\chi}_{2}^{+}} \\
& +2\left(k_{11}^{\tilde{t}} k_{12}^{\tilde{t}} Q_{11}^{L \prime} Q_{12}^{L \prime}+l_{11}^{\tilde{t}} l_{12}^{\tilde{t}} Q_{11}^{R \prime} Q_{12}^{R \prime}\right) m_{\tilde{\chi}_{1}^{+}} m_{\tilde{\chi}_{2}^{+}}, \\
& a_{33}=2\left(l_{11}^{\tilde{t}} l_{12}^{\tilde{t}} Q_{12}^{L \prime} Q_{11}^{R \prime}+k_{11}^{\tilde{t}} k_{12}^{\tilde{t}} Q_{11}^{L \prime} Q_{12}^{R \prime}\right) m_{\tilde{\chi}_{1}^{0}} m_{\tilde{\chi}_{1}^{+}} \\
& +4\left(k_{11}^{\tilde{t}} l_{12}^{\tilde{t}} Q_{12}^{L \prime} Q_{11}^{R \prime}+k_{12}^{\tilde{t}} l_{11}^{\tilde{t}} Q_{11}^{L \prime} Q_{12}^{R \prime}\right) m_{b} m_{\tilde{\chi}_{1}^{0}} \\
& +2\left(k_{11}^{\tilde{t}} k_{12}^{\tilde{t}} Q_{12}^{L \prime} Q_{11}^{R \prime}+l_{11}^{\tilde{t}} l_{12}^{\tilde{t}} Q_{11}^{L \prime} Q_{12}^{R \prime}\right) m_{\tilde{\chi}_{1}^{0}} m_{\tilde{\chi}_{2}^{+}} \\
& +2\left(l_{11}^{\tilde{t}} l_{12}^{\tilde{t}} Q_{11}^{L \prime} Q_{12}^{L \prime}+k_{11}^{\tilde{t}} k_{12}^{\tilde{t}} Q_{11}^{R \prime} Q_{12}^{R \prime}\right)\left(2 m_{b}^{2}+2 m_{\tilde{\chi}_{1}^{0}}^{2}+m_{H^{+}}^{2}+m_{\tilde{t}_{1}}^{2}\right) \\
& +2\left(k_{11}^{\tilde{t}} l_{12}^{\tilde{t}} Q_{11}^{L \prime} Q_{12}^{L \prime}+k_{12}^{\tilde{t}} l_{11}^{\tilde{t}} Q_{11}^{R \prime} Q_{12}^{R \prime}\right) m_{b} m_{\tilde{\chi}_{1}^{+}} \\
& +2\left(k_{12}^{\tilde{t}} l_{11}^{\tilde{t}} Q_{11}^{L \prime} Q_{12}^{L \prime}+k_{11}^{\tilde{t}} l_{12}^{\tilde{t}} Q_{11}^{R \prime} Q_{12}^{R \prime}\right) m_{b} m_{\tilde{\chi}_{2}^{+}} \text {, } \\
& a_{34}=-2\left(l_{11}^{\tilde{t}} l_{12}^{\tilde{t}} Q_{11}^{L \prime} Q_{12}^{L \prime}+k_{11}^{\tilde{t}} k_{12}^{\tilde{t}} Q_{11}^{R \prime} Q_{12}^{R \prime}\right) \text {, } \\
& b_{11}=\frac{\sqrt{2}}{m_{W}}\left\{b_{11}^{\tilde{t}} k_{11}^{\tilde{t}} Q_{11}^{L \prime} m_{\tilde{\chi}_{1}^{+}} m_{b} m_{t}\left[\left(m_{\tilde{t}_{1}}^{2}-m_{\tilde{\chi}_{1}^{0}}^{2}\right) \cot \beta-\left(m_{b}^{2}+m_{\tilde{\chi}_{1}^{0}}^{2}\right) \tan \beta\right]\right. \\
& +b_{11}^{\tilde{t}} l_{11}^{\tilde{t}} Q_{11}^{L}{ }^{\prime} m_{t}\left[\left(m_{H^{+}}^{2} m_{\tilde{t}_{1}}^{2}-m_{b}^{2} m_{\tilde{\chi}_{1}^{0}}^{2}\right) \cot \beta-m_{b}^{2}\left(m_{b}^{2}+m_{\tilde{t}_{1}}^{2}+2 m_{\tilde{\chi}_{1}^{0}}^{2}\right) \tan \beta\right] \\
& +a_{11}^{\tilde{t}} l_{11}^{\tilde{t}} Q_{11}^{L \prime} m_{\tilde{\chi}_{1}^{0}} \\
& *\left[m_{b}^{2}\left(m_{H^{+}}^{2}+m_{\tilde{t}_{1}}^{2}-m_{\tilde{\chi}_{1}^{0}}^{2}-m_{b}^{2}\right) \tan \beta-m_{t}^{2}\left(2 m_{b}^{2}+m_{\tilde{\chi}_{1}^{0}}^{2}+m_{H^{+}}^{2}\right) \cot \beta\right] \\
& +a_{11}^{\tilde{t}} k_{11}^{\tilde{t}} Q_{11}^{L \prime} m_{\tilde{\chi}_{1}^{+}} m_{b} m_{\tilde{\chi}_{1}^{0}}\left[\left(m_{H^{+}}^{2}-m_{b}^{2}\right) \tan \beta-2 m_{t}^{2} \cot \beta\right] \\
& +b_{11}^{\tilde{t}} k_{11}^{\tilde{t}} Q_{11}^{R \prime} m_{\tilde{\chi}_{1}^{0}} m_{b} m_{t} \\
& *\left[\left(m_{\tilde{t}_{1}}^{2}+m_{H^{+}}^{2}-m_{b}^{2}-m_{\tilde{\chi}_{1}^{0}}^{2}\right) \cot \beta-\left(m_{H^{+}}^{2}+2 m_{b}^{2}+m_{\tilde{\chi}_{1}^{0}}^{2}\right) \tan \beta\right] \\
& +b_{11}^{\tilde{t}} l_{11}^{\tilde{t}} Q_{11}^{R \prime} m_{t} m_{\tilde{\chi}_{1}^{0}} m_{\tilde{\chi}_{1}^{+}}\left[\left(m_{H^{+}}^{2}-m_{b}^{2}\right) \cot \beta-2 m_{b}^{2} \tan \beta\right] \\
& -a_{11}^{\tilde{t}} k_{11}^{\tilde{t}} Q_{11}^{R \prime} m_{b}
\end{aligned}
$$




$$
\begin{aligned}
& *\left[\left(m_{b}^{2} m_{\tilde{\chi}_{1}^{0}}^{2}-m_{\tilde{t}_{1}}^{2} m_{H^{+}}^{2}\right) \tan \beta+m_{t}^{2}\left(m_{b}^{2}+m_{\tilde{t}_{1}}^{2}+2 m_{\tilde{\chi}_{1}^{0}}^{2}\right) \cot \beta\right] \\
& \left.-a_{11}^{\tilde{t}} l_{11}^{\tilde{t}} Q_{11}^{R \prime} m_{\tilde{\chi}_{1}^{+}}\left[m_{b}^{2}\left(m_{\tilde{\chi}_{1}^{0}}^{2}-m_{\tilde{t}_{1}}^{2}\right) \tan \beta+m_{t}^{2}\left(m_{b}^{2}+m_{\tilde{\chi}_{1}^{0}}^{2}\right) \cot \beta\right]\right\} \text {, } \\
& b_{12}=\frac{\sqrt{2}}{m_{W}}\left\{b_{11}^{\tilde{t}} k_{11}^{\tilde{t}} Q_{11}^{L \prime} m_{\tilde{\chi}_{1}^{+}} m_{b} m_{t} \tan \beta+b_{11}^{\tilde{t}} l_{11}^{\tilde{t}} Q_{11}^{L}{ }^{\prime} m_{b}^{2} m_{t} \tan \beta\right. \\
& +a_{11}^{\tilde{t}} l_{11}^{\tilde{t}} Q_{11}^{L \prime} m_{t}^{2} m_{\tilde{\chi}_{1}^{0}} \cot \beta+b_{11}^{\tilde{t}} k_{11}^{\tilde{t}} Q_{11}^{R \prime} m_{\tilde{\chi}_{1}^{0}} m_{b} m_{t} \tan \beta \\
& \left.+a_{11}^{\tilde{t}} l_{11}^{\tilde{t}} Q_{11}^{R \prime} m_{\tilde{\chi}_{1}^{+}} m_{t}^{2} \cot \beta+a_{11}^{\tilde{t}} k_{11}^{\tilde{t}} Q_{11}^{R \prime} m_{b} m_{t}^{2} \cot \beta\right\} \text {, } \\
& b_{13}=-\frac{\sqrt{2}}{m_{W}}\left\{b_{11}^{\tilde{t}} k_{11}^{\tilde{t}} Q_{11}^{L \prime} m_{\tilde{\chi}_{1}^{+}} m_{b} m_{t} \cot \beta-a_{11}^{\tilde{t}} l_{11}^{\tilde{t}} Q_{11}^{L \prime} m_{t}^{2} m_{\tilde{\chi}_{1}^{0}} \cot \beta\right. \\
& +b_{11}^{\tilde{t}} l_{11}^{\tilde{t}} Q_{11}^{L \prime} m_{t}\left[\left(m_{\tilde{\chi}_{1}^{0}}^{2}+m_{H^{+}}^{2}+m_{\tilde{t}_{1}}^{2}+m_{b}^{2}\right) \cot \beta-m_{b}^{2} \tan \beta\right] \\
& +a_{11}^{\tilde{t}} k_{11}^{\tilde{t}} Q_{11}^{L \prime} m_{\tilde{\chi}_{1}^{+}} m_{b} m_{\tilde{\chi}_{1}^{0}} \tan \beta-b_{11}^{\tilde{t}} k_{11}^{\tilde{t}} Q_{11}^{R \prime} m_{\tilde{\chi}_{1}^{0}} m_{b} m_{t} \tan \beta \\
& +b_{11}^{\tilde{t}} l_{11}^{\tilde{t}} Q_{11}^{R \prime} m_{\tilde{\chi}_{1}^{+}} m_{t} m_{\tilde{\chi}_{1}^{0}} \cot \beta+a_{11}^{\tilde{t}} l_{11}^{\tilde{t}} Q_{11}^{R \prime} m_{b}^{2} m_{\tilde{\chi}_{1}^{+}} \tan \beta \\
& \left.+a_{11}^{\tilde{t}} k_{11}^{\tilde{t}} Q_{11}^{R \prime} m_{b}\left[\left(m_{b}^{2}+m_{\tilde{\chi}_{1}^{0}}^{2}+m_{H^{+}}^{2}+m_{\tilde{t}_{1}}^{2}\right) \tan \beta-m_{t}^{2} \cot \beta\right]\right\} \text {, } \\
& b_{14}=\frac{\sqrt{2}}{m_{W}}\left(b_{11}^{\tilde{t}} l_{11}^{\tilde{t}} Q_{11}^{L \prime} m_{t} \cot \beta+a_{11}^{\tilde{t}} k_{11}^{\tilde{t}} Q_{11}^{R \prime} m_{b} \tan \beta\right) \text {, } \\
& c_{111}=2 C_{\tilde{t}_{1} \tilde{b}_{1}}^{H}\left[\left(b_{11}^{\tilde{b}} l_{11}^{\tilde{t}} Q_{11}^{L \prime}+a_{11}^{\tilde{b}} k_{11}^{\tilde{t}} Q_{11}^{R \prime}\right) m_{b}\left(m_{b}^{2}+m_{\tilde{t}_{1}}^{2}+2 m_{\tilde{\chi}_{1}^{0}}^{2}\right)\right. \\
& +\left(a_{11}^{\tilde{b}} l_{11}^{\tilde{t}} Q_{11}^{L \prime}+b_{11}^{\tilde{b}} k_{11}^{\tilde{t}} Q_{11}^{R \prime}\right) m_{\tilde{\chi}_{1}^{0}}\left(m_{\tilde{\chi}_{1}^{0}}^{2}+m_{H^{+}}^{2}+2 m_{b}^{2}\right) \\
& +\left(b_{11}^{\tilde{b}} k_{11}^{\tilde{t}} Q_{11}^{L \prime}+a_{11}^{\tilde{b}} l_{11}^{\tilde{t}} Q_{11}^{R \prime}\right) m_{\tilde{\chi}_{1}^{+}}\left(m_{b}^{2}+m_{\tilde{\chi}_{1}^{0}}^{2}\right) \\
& \left.+\left(a_{11}^{\tilde{b}} k_{11}^{\tilde{t}} Q_{11}^{L \prime}+b_{11}^{\tilde{b}} l_{11}^{\tilde{t}} Q_{11}^{R \prime}\right) 2 m_{b} m_{\tilde{\chi}_{1}^{0}} m_{\tilde{\chi}_{1}^{+}}\right], \\
& c_{112}=-2 C_{\tilde{t}_{1} \tilde{b}_{1}}^{H}\left[\left(b_{11}^{\tilde{b}} l_{11}^{\tilde{t}} Q_{11}^{L \prime}+a_{11}^{\tilde{b}} k_{11}^{\tilde{t}} Q_{11}^{R \prime}\right) m_{b}+\left(a_{11}^{\tilde{b}} l_{11}^{\tilde{t}} Q_{11}^{L \prime}+b_{11}^{\tilde{b}} k_{11}^{\tilde{t}} Q_{11}^{R \prime}\right) m_{\tilde{\chi}_{1}^{0}}\right. \\
& \left.+\left(b_{11}^{\tilde{b}} k_{11}^{\tilde{t}} Q_{11}^{L \prime}+a_{11}^{\tilde{b}} l_{11}^{\tilde{t}} Q_{11}^{R \prime}\right) m_{\tilde{\chi}_{1}^{+}}\right], \\
& c_{113}=-2 C_{\tilde{t}_{1} \tilde{b}_{1}}^{H}\left[\left(b_{11}^{\tilde{b}} l_{11}^{\tilde{t}} Q_{11}^{L \prime}+a_{11}^{\tilde{b}} k_{11}^{\tilde{t}} Q_{11}^{R \prime}\right) m_{b}+\left(a_{11}^{\tilde{b}} l_{11}^{\tilde{t}} Q_{11}^{L \prime}+b_{11}^{\tilde{b}} k_{11}^{\tilde{t}} Q_{11}^{R \prime}\right) m_{\tilde{\chi}_{1}^{0}}\right] \text {, } \\
& d_{1}=\frac{1}{2 m_{W}^{2}}\left[-\left(\left(a_{11}^{\tilde{t}}\right)^{2} m_{t}^{2} \cot ^{2} \beta+\left(b_{11}^{\tilde{t}}\right)^{2} m_{b}^{2} \tan ^{2} \beta\right) m_{t}^{2}\left(m_{b}^{2}+m_{\tilde{\chi}_{1}^{0}}^{2}\right)\right. \\
& +\left(\left(a_{11}^{\tilde{t}}\right)^{2} m_{b}^{2} \tan ^{2} \beta+\left(b_{11}^{\tilde{t}}\right)^{2} m_{t}^{2} \cot ^{2} \beta\right)\left(m_{\tilde{\chi}_{1}^{0}}^{2}-m_{\tilde{t}_{1}}^{2}\right)\left(m_{H^{+}}^{2}-m_{b}^{2}\right) \\
& +2 a_{11}^{\tilde{t}} b_{11}^{\tilde{t}} m_{t} m_{\tilde{\chi}_{1}^{0}}\left[\left(m_{H^{+}}^{2}-m_{b}^{2}\right)\left(m_{b}^{2} \tan ^{2} \beta+m_{t}^{2} \cot ^{2} \beta\right)-2 m_{b}^{2} m_{t}^{2}\right]
\end{aligned}
$$




$$
\begin{aligned}
& \left.+2\left(\left(a_{11}^{\tilde{t}}\right)^{2}+\left(b_{11}^{\tilde{t}}\right)^{2}\right) m_{b}^{2} m_{t}^{2}\left(m_{\tilde{t}_{1}}^{2}-m_{\tilde{\chi}_{1}^{0}}^{2}\right)\right] \\
& d_{2}=\frac{m_{t}^{2}}{2 m_{W}^{2}}\left(\left(a_{11}^{\tilde{t}}\right)^{2} m_{t}^{2} \cot ^{2} \beta+\left(b_{11}^{\tilde{t}}\right)^{2} m_{b}^{2} \tan ^{2} \beta\right) \text {, } \\
& d_{3}=\frac{-1}{2 m_{W}^{2}}\left[2\left(\left(a_{11}^{\tilde{t}}\right)^{2}+\left(b_{11}^{\tilde{t}}\right)^{2}\right) m_{b}^{2} m_{t}^{2}+2 a_{11}^{\tilde{t}} b_{11}^{\tilde{t}} m_{t} m_{\tilde{\chi}_{1}^{0}}\left(m_{b}^{2}\left(2+\tan ^{2} \beta\right)+m_{t}^{2} \cot ^{2} \beta\right)\right. \\
& \left.-\left(\left(a_{11}^{\tilde{t}}\right)^{2} m_{b}^{2} \tan ^{2} \beta+\left(b_{11}^{\tilde{t}}\right)^{2} m_{t}^{2} \cot ^{2} \beta\right)\left(m_{H^{+}}^{2}+m_{\tilde{t}_{1}}^{2}\right)\right], \\
& d_{4}=-\frac{\left(a_{11}^{\tilde{t}}\right)^{2} m_{b}^{2} \tan ^{2} \beta+\left(b_{11}^{\tilde{t}}\right)^{2} m_{t}^{2} \cot ^{2} \beta}{2 m_{W}^{2}}, \\
& e_{11}=-\frac{\sqrt{2} C_{\tilde{t}_{1} \tilde{b}_{1}}^{H}}{m_{W}}\left\{a_{11}^{\tilde{b}} b_{11}^{\tilde{t}} m_{t} m_{\tilde{\chi}_{1}^{0}}\left[\left(m_{H^{+}}^{2}-m_{b}^{2}\right) \cot \beta-2 m_{b}^{2} \tan \beta\right]\right. \\
& +b_{11}^{\tilde{b}} b_{11}^{\tilde{t}} m_{b} m_{t}\left[\left(m_{\tilde{t}_{1}}^{2}-m_{\tilde{\chi}_{1}^{0}}^{2}\right) \cot \beta-\left(m_{b}^{2}+m_{\tilde{\chi}_{1}^{0}}^{2}\right) \tan \beta\right] \\
& -a_{11}^{\tilde{b}} a_{11}^{\tilde{t}}\left[m_{t}^{2}\left(m_{b}^{2}+m_{\tilde{\chi}_{1}^{0}}^{2}\right) \cot \beta+m_{b}^{2}\left(m_{\tilde{\chi}_{1}^{0}}^{2}-m_{\tilde{t}_{1}}^{2}\right) \tan \beta\right] \\
& \left.-b_{11}^{\tilde{b}} a_{11}^{\tilde{t}} m_{b} m_{\tilde{\chi}_{1}^{0}}\left[2 m_{t}^{2} \cot \beta+\left(m_{b}^{2}-m_{H^{+}}^{2}\right) \tan \beta\right]\right\} \\
& e_{12}=-\frac{\sqrt{2} C_{\tilde{t}_{1} \tilde{b}_{1}}^{H}}{m_{W}}\left(a_{11}^{\tilde{b}} a_{11}^{\tilde{t}} m_{t}^{2} \cot \beta+b_{11}^{\tilde{b}} b_{11}^{\tilde{t}} m_{b} m_{t} \tan \beta\right), \\
& e_{13}=\frac{\sqrt{2} C_{\tilde{t}_{1} \tilde{b}_{1}}^{H}}{m_{W}}\left(a_{11}^{\tilde{b}} b_{11}^{\tilde{t}} m_{t} m_{\tilde{\chi}_{1}^{0}} \cot \beta+b_{11}^{\tilde{b}} b_{11}^{\tilde{t}} m_{b} m_{t} \cot \beta\right. \\
& \left.+a_{11}^{\tilde{b}} a_{11}^{\tilde{t}} m_{b}^{2} \tan \beta+b_{11}^{\tilde{b}} a_{11}^{\tilde{t}} m_{b} m_{\tilde{\chi}_{1}^{0}} \tan \beta\right), \\
& f_{11}=-\left(C_{\tilde{t}_{1} \tilde{b}_{1}}^{H}\right)^{2}\left[\left(\left(a_{11}^{\tilde{b}}\right)^{2}+\left(b_{11}^{\tilde{b}}\right)^{2}\right)\left(m_{b}^{2}+m_{\tilde{\chi}_{1}^{0}}^{2}\right)+4 a_{11}^{\tilde{b}} b_{11}^{\tilde{b}} m_{b} m_{\tilde{\chi}_{1}^{0}}\right], \\
& f_{12}=\left(C_{\tilde{t}_{1} \tilde{b}_{1}}^{H}\right)^{2}\left(\left(a_{11}^{\tilde{b}}\right)^{2}+\left(b_{11}^{\tilde{b}}\right)^{2}\right) \text {, } \\
& f_{31}=-2 C_{\tilde{t}_{1} \tilde{b}_{1}}^{H} C_{\tilde{t}_{1} \tilde{b}_{2}}^{H}\left[\left(a_{11}^{\tilde{b}} a_{12}^{\tilde{b}}+b_{11}^{\tilde{b}} b_{12}^{\tilde{b}}\right)\left(m_{b}^{2}+m_{\tilde{\chi}_{1}^{0}}^{2}\right)+2\left(a_{11}^{\tilde{b}} b_{12}^{\tilde{b}}+b_{11}^{\tilde{b}} a_{12}^{\tilde{b}}\right) m_{b} m_{\tilde{\chi}_{1}^{0}}\right], \\
& f_{32}=2 C_{\tilde{t}_{1} \tilde{b}_{1}}^{H} C_{\tilde{t}_{1} \tilde{b}_{2}}^{H}\left(a_{11}^{\tilde{b}} a_{12}^{\tilde{b}}+b_{11}^{\tilde{b}} b_{12}^{\tilde{b}}\right) .
\end{aligned}
$$


One gets the remaining coefficients by replacements:

$a_{1 i} \rightarrow a_{2 i}: \quad l_{\tilde{\tau}_{11}}^{\tilde{t}} \rightarrow l_{\tilde{1}}^{\tilde{t}}, k_{11}^{\tilde{t}} \rightarrow k_{\tilde{\tau}}^{\tilde{t}}, Q_{11}^{L \prime} \rightarrow Q_{12}^{L \prime}, Q_{11}^{R \prime} \rightarrow Q_{12}^{R \prime}, m_{\tilde{\chi}_{1}^{+}} \rightarrow m_{\tilde{\chi}_{2}^{+}}$

$b_{1 i} \rightarrow b_{2 i}: \quad l_{11}^{\tilde{t}} \rightarrow l_{12}^{\tilde{t}}, k_{11}^{\tilde{t}} \rightarrow k_{12}^{\tilde{t}}, Q_{11}^{L \prime} \rightarrow Q_{12}^{L \prime}, Q_{11}^{R \prime} \rightarrow Q_{12}^{R \prime}, m_{\tilde{\chi}_{1}^{+}} \rightarrow m_{\tilde{\chi}_{2}^{+}}$

$c_{11 i} \rightarrow c_{12 i}: l_{11}^{\tilde{t}} \rightarrow l_{12}^{\tilde{t}}, k_{11}^{\tilde{t}} \rightarrow k_{12}^{\tilde{t}}, Q_{11}^{L \prime} \rightarrow Q_{12}^{L \prime}, Q_{11}^{R \prime} \rightarrow Q_{12}^{R \prime}, m_{\tilde{\chi}_{1}^{+}} \rightarrow m_{\tilde{\chi}_{2}^{+}}$

$c_{11 i} \rightarrow c_{21 i}: a_{11}^{\tilde{b}} \rightarrow a_{12}^{\tilde{b}}, b_{11}^{\tilde{b}} \rightarrow b_{12}^{\tilde{b}}, m_{\tilde{b}_{1}} \rightarrow m_{\tilde{b}_{2}}, C_{\tilde{t}_{1} \tilde{b}_{1}}^{H} \rightarrow C_{\tilde{t}_{1} \tilde{b}_{2}}^{H}$

$c_{11 i} \rightarrow c_{22 i}: l_{11}^{\tilde{t}} \rightarrow l_{12}^{\tilde{t}}, k_{11}^{\tilde{t}} \rightarrow k_{\tilde{\tilde{t}}}^{\tilde{t}}, Q_{11}^{L \prime} \rightarrow Q_{12}^{L \prime}{ }^{L}, Q_{11}^{R \prime} \rightarrow Q_{12}^{R \prime}, m_{\tilde{\chi}_{1}^{+}} \rightarrow m_{\tilde{\chi}_{2}^{+}}$,

$a_{11}^{\tilde{b}} \rightarrow a_{12}^{\tilde{b}}, b_{11}^{\tilde{b}} \rightarrow b_{12}^{\tilde{b}}, m_{\tilde{b}_{1}} \rightarrow m_{\tilde{b}_{2}}, C_{\tilde{t}_{1} \tilde{b}_{1}}^{H} \rightarrow C_{\tilde{t}_{1} \tilde{b}_{2}}^{H}$

$e_{1 i} \rightarrow e_{2 i}: \quad a_{11}^{\tilde{b}} \rightarrow a_{12}^{\tilde{b}}, b_{11}^{\tilde{b}} \rightarrow b_{12}^{\tilde{b}}, m_{\tilde{b}_{1}} \rightarrow m_{\tilde{b}_{2}}, C_{\tilde{t}_{1} \tilde{b}_{1}}^{H} \rightarrow C_{\tilde{t}_{1} \tilde{b}_{2}}^{H}$

$f_{1 i} \rightarrow f_{2 i}: \quad a_{11}^{\tilde{b}} \rightarrow a_{12}^{\tilde{b}}, b_{11}^{\tilde{b}} \rightarrow b_{12}^{\tilde{b}}, m_{\tilde{b}_{1}} \rightarrow m_{\tilde{b}_{2}}, C_{\tilde{t}_{1} \tilde{b}_{1}}^{H} \rightarrow C_{\tilde{t}_{1} \tilde{b}_{2}}^{H}$

\section{The widths $\Gamma\left(\tilde{t}_{1} \rightarrow b \tilde{l} l^{\prime}\right)$}

Here the decay width is given by

$$
\Gamma\left(\tilde{t}_{1} \rightarrow b \tilde{l} l^{\prime}\right)=\frac{\alpha^{2}}{16 \pi m_{\tilde{t}_{1}}^{3} \sin ^{4} \theta_{W}} \int_{\left(m_{l^{\prime}}+m_{\tilde{l}}\right)^{2}}^{\left(m_{\tilde{t}_{1}}-m_{b}\right)^{2}} d s W_{l^{\prime} \tilde{l}}(s) \sum_{i=1}^{3}\left(\sum_{j=1}^{5} c_{i j} s^{(j-4)}\right) D_{i}(s)
$$

with

$$
\begin{aligned}
D_{1,2}(s) & =\frac{1}{\left(s-m_{\tilde{\chi}_{1,2}^{+}}^{2}\right)^{2}+m_{\tilde{\chi}_{1,2}^{+}}^{2} \Gamma_{\tilde{\chi}_{1,2}^{+}}^{2}}, \\
D_{3}(s) & =\operatorname{Re}\left(\frac{1}{\left(s-m_{\tilde{\chi}_{1}^{+}}^{2}+i m_{\tilde{\chi}_{1}^{+}} \Gamma_{\tilde{\chi}_{1}^{+}}\right)\left(s-m_{\tilde{\chi}_{2}^{+}}^{2}-i m_{\tilde{\chi}_{2}^{+}} \Gamma_{\tilde{\chi}_{2}^{+}}\right)}\right) .
\end{aligned}
$$

In the case of $\tilde{t}_{1} \rightarrow b \tilde{\nu}_{e} e^{+}$one finds in the limit $m_{e} \rightarrow 0$ that

$$
\begin{aligned}
& W_{e \tilde{\nu}_{e}}(s)=\lambda^{\frac{1}{2}}\left(s, m_{\tilde{t}_{1}}^{2}, m_{b}^{2}\right)\left(s-m_{\tilde{\nu}_{e}}^{2}\right), \\
& c_{11}=\frac{1}{2}\left(k_{11}^{\tilde{t}}\right)^{2} V_{11}^{2} m_{\tilde{\chi}_{1}^{+}}^{2} m_{\tilde{\nu}_{e}}^{2}\left(m_{b}^{2}-m_{\tilde{t}_{1}}^{2}\right), \\
& c_{12}=V_{11}^{2}\left[\frac{1}{2}\left(l_{11}^{\tilde{t}}\right)^{2} m_{\tilde{\nu}_{e}}^{2}\left(m_{b}^{2}-m_{\tilde{t}_{1}}^{2}\right)+\frac{1}{2}\left(k_{11}^{\tilde{t}}\right)^{2} m_{\tilde{\chi}_{1}^{+}}^{2}\left(m_{\tilde{t}_{1}}^{2}+m_{\tilde{\nu}_{e}}^{2}-m_{b}^{2}\right)\right. \\
& \left.+2 k_{11}^{\tilde{t}} l_{11}^{\tilde{t}} m_{b} m_{\tilde{\chi}_{1}^{+}} m_{\tilde{\nu}_{e}}^{2}\right] \text {, } \\
& c_{13}=V_{11}^{2}\left[\frac{1}{2}\left(l_{11}^{\tilde{t}}\right)^{2}\left(m_{\tilde{t}_{1}}^{2}+m_{\tilde{\nu}_{e}}^{2}-m_{b}^{2}\right)-2 k_{11}^{\tilde{t}} l_{11}^{\tilde{t}} m_{b} m_{\tilde{\chi}_{1}^{+}}-\frac{1}{2}\left(k_{11}^{\tilde{t}}\right)^{2} m_{\tilde{\chi}_{1}^{+}}^{2}\right] \text {, } \\
& c_{14}=-\frac{\left(l_{11}^{\tilde{t}}\right)^{2} V_{11}^{2}}{2} \\
& c_{31}=k_{11}^{\tilde{t}} k_{12}^{\tilde{t}} V_{11} V_{12} m_{\tilde{\chi}_{1}^{+}} m_{\tilde{\chi}_{2}^{+}}\left(m_{b}^{2}-m_{\tilde{t}_{1}}^{2}\right) m_{\tilde{\nu}_{e}}^{2}, \\
& c_{32}=V_{11} V_{12}\left[l_{11}^{\tilde{t}} l_{12}^{\tilde{t}} m_{\tilde{\nu}_{e}}^{2}\left(m_{b}^{2}-m_{\tilde{t}_{1}}^{2}\right)+k_{11}^{\tilde{t}} k_{12}^{\tilde{t}} m_{\tilde{\chi}_{1}^{+}} m_{\tilde{\chi}_{2}^{+}}\left(m_{\tilde{t}_{1}}^{2}+m_{\tilde{\nu}_{e}}^{2}-m_{b}^{2}\right)\right.
\end{aligned}
$$




$$
\begin{gathered}
\left.+2 k_{11}^{\tilde{t}} l_{12}^{\tilde{t}} m_{b} m_{\tilde{\chi}_{1}^{+}} m_{\tilde{\nu}_{e}}^{2}+2 k_{12}^{\tilde{t}} l_{11}^{\tilde{t}} m_{b} m_{\tilde{\chi}_{2}^{+}} m_{\tilde{\nu}_{e}}^{2}\right], \\
c_{33}=V_{11} V_{12}\left[l_{11}^{\tilde{t}} l_{12}^{\tilde{t}}\left(m_{\tilde{t}_{1}}^{2}+m_{\tilde{\nu}_{e}}^{2}-m_{b}^{2}\right)-2 k_{11}^{\tilde{t}} l_{12}^{\tilde{t}} m_{b} m_{\tilde{\chi}_{1}^{+}}\right. \\
\left.\quad-2 k_{12}^{\tilde{t}} l_{11}^{\tilde{t}} m_{b} m_{\tilde{\chi}_{2}^{+}}-k_{11}^{\tilde{t}} k_{12}^{\tilde{t}} m_{\tilde{\chi}_{1}^{+}} m_{\tilde{\chi}_{2}^{+}}\right], \\
c_{34}=-l_{11}^{\tilde{t}} l_{12}^{\tilde{t}} V_{11} V_{12}, \\
c_{15}=c_{25}=c_{35}=0 .
\end{gathered}
$$

The coefficients $c_{2 i}$ are obtained from $c_{1 i}$ by the replacements: $k_{11}^{\tilde{t}} \rightarrow k_{12}^{\tilde{t}}, l_{11}^{\tilde{t}} \rightarrow l_{12}^{\tilde{t}}, V_{11} \rightarrow V_{12}$ and $m_{\tilde{\chi}_{1}^{+}} \rightarrow m_{\tilde{\chi}_{2}^{+}}$. In the case of $\tilde{t}_{1} \rightarrow b \tilde{\nu}_{\tau} \tau^{+}$one finds that

$$
\begin{aligned}
& W_{\tau \tilde{\nu}_{\tau}}(s)=\lambda^{\frac{1}{2}}\left(s, m_{\tilde{t}_{1}}^{2}, m_{b}^{2}\right) \lambda^{\frac{1}{2}}\left(s, m_{\tilde{\nu}_{\tau}}^{2}, m_{\tau}^{2}\right) \text {, } \\
& c_{11}=\frac{1}{2}\left(\left(k_{1}^{\tilde{\nu}_{\tau}}\right)^{2}\left(l_{11}^{\tilde{t}}\right)^{2}+\left(l_{1}^{\tilde{\nu}_{\tau}}\right)^{2}\left(k_{11}^{\tilde{t}}\right)^{2}\right) m_{\tilde{\chi}_{1}^{+}}^{2}\left(m_{b}^{2}-m_{\tilde{t}_{1}}^{2}\right)\left(m_{\tilde{\nu}_{\tau}}^{2}-m_{\tau}^{2}\right), \\
& c_{12}=\frac{1}{2}\left(\left(k_{1}^{\tilde{\nu}_{\tau}}\right)^{2}\left(k_{11}^{\tilde{t}}\right)^{2}+\left(l_{1}^{\tilde{\nu}_{\tau}}\right)^{2}\left(l_{11}^{\tilde{t}}\right)^{2}\right)\left(m_{\tilde{t}_{1}}^{2}-m_{b}^{2}\right)\left(m_{\tau}^{2}-m_{\tilde{\nu}_{\tau}}^{2}\right) \\
& +\frac{1}{2}\left(\left(k_{1}^{\tilde{\nu}_{\tau}}\right)^{2}\left(l_{11}^{\tilde{t}}\right)^{2}+\left(l_{1}^{\tilde{\nu}_{\tau}}\right)^{2}\left(k_{11}^{\tilde{t}}\right)^{2}\right) m_{\tilde{\chi}_{1}^{+}}^{2}\left(m_{\tilde{t}_{1}}^{2}+m_{\tilde{\nu}_{\tau}}^{2}-m_{b}^{2}-m_{\tau}^{2}\right) \\
& +2 k_{1}^{\tilde{\nu}_{\tau}} l_{1}^{\tilde{\nu}_{\tau}}\left(\left(k_{11}^{\tilde{t}}\right)^{2}+\left(l_{11}^{\tilde{t}}\right)^{2}\right) m_{\tau} m_{\tilde{\chi}_{1}^{+}}\left(m_{\tilde{t}_{1}}^{2}-m_{b}^{2}\right) \\
& +2 k_{11}^{\tilde{t}} l_{11}^{\tilde{t}}\left(\left(k_{1}^{\tilde{\nu}_{\tau}}\right)^{2}+\left(l_{1}^{\tilde{\nu}_{\tau}}\right)^{2}\right) m_{b} m_{\tilde{\chi}_{1}^{+}}\left(m_{\tilde{\nu}_{\tau}}^{2}-m_{\tau}^{2}\right)-4 k_{1}^{\tilde{\nu}_{\tau}} l_{1}^{\tilde{\nu}_{\tau}} k_{11}^{\tilde{t}} l_{11}^{\tilde{t}} m_{b} m_{\tau} m_{\tilde{\chi}_{1}^{+}}^{2} \text {, } \\
& c_{13}=\frac{1}{2}\left(\left(k_{1}^{\tilde{\nu}_{\tau}}\right)^{2}\left(k_{11}^{\tilde{t}}\right)^{2}+\left(l_{1}^{\tilde{\nu}_{\tau}}\right)^{2}\left(l_{11}^{\tilde{t}}\right)^{2}\right)\left(m_{\tilde{t}_{1}}^{2}+m_{\tilde{\nu}_{\tau}}^{2}-m_{b}^{2}-m_{\tau}^{2}\right) \\
& -2 k_{11}^{\tilde{t}} l_{11}^{\tilde{t}}\left(\left(k_{1}^{\tilde{\nu}_{\tau}}\right)^{2}+\left(l_{1}^{\tilde{\nu}_{\tau}}\right)^{2}\right) m_{b} m_{\tilde{\chi}_{1}^{+}}-\frac{1}{2}\left(\left(k_{1}^{\tilde{\nu}_{\tau}}\right)^{2}\left(l_{11}^{\tilde{t}}\right)^{2}+\left(l_{1}^{\tilde{\nu}_{\tau}}\right)^{2}\left(k_{11}^{\tilde{t}}\right)^{2}\right) m_{\tilde{\chi}_{1}^{+}}^{2} \\
& -4 k_{1}^{\tilde{\nu}_{\tau}} l_{1}^{\tilde{\nu}_{\tau}} k_{11}^{\tilde{t}} l_{11}^{\tilde{t}} m_{b} m_{\tau}-2 k_{1}^{\tilde{\nu}_{\tau}} l_{1}^{\tilde{\nu}_{\tau}}\left(\left(k_{11}^{\tilde{t}}\right)^{2}+\left(l_{11}^{\tilde{t}}\right)^{2}\right) m_{\tau} m_{\tilde{\chi}_{1}^{+}} \text {, } \\
& c_{14}=-\frac{1}{2}\left(\left(k_{1}^{\tilde{\nu}_{\tau}}\right)^{2}\left(k_{11}^{\tilde{t}}\right)^{2}+\left(l_{1}^{\tilde{\nu}_{\tau}}\right)^{2}\left(l_{11}^{\tilde{t}}\right)^{2}\right) \text {, } \\
& c_{31}=\left(k_{1}^{\tilde{\nu}_{\tau}} k_{2}^{\tilde{\nu}_{\tau}} l_{11}^{\tilde{t}} l_{12}^{\tilde{t}}+k_{11}^{\tilde{t}} k_{12}^{\tilde{t}} l_{1}^{\tilde{\nu}_{\tau}} l_{2}^{\tilde{\nu}_{\tau}}\right) m_{\tilde{\chi}_{1}^{+}} m_{\tilde{\chi}_{2}^{+}}\left(m_{b}^{2}-m_{\tilde{t}_{1}}^{2}\right)\left(m_{\tilde{\nu}_{\tau}}^{2}-m_{\tau}^{2}\right) \text {, } \\
& c_{32}=\left(k_{1}^{\tilde{\nu}_{\tau}} k_{2}^{\tilde{\nu}_{\tau}} k_{11}^{\tilde{t}} k_{12}^{\tilde{t}}+l_{1}^{\tilde{\nu}_{\tau}} l_{2}^{\tilde{\nu}_{\tau}} l_{11}^{\tilde{t}} l_{12}^{\tilde{t}}\right)\left(m_{\tilde{t}_{1}}^{2}-m_{b}^{2}\right)\left(m_{\tau}^{2}-m_{\tilde{\nu}_{\tau}}^{2}\right) \\
& +\left(k_{1}^{\tilde{\nu}_{\tau}} k_{2}^{\tilde{\nu}_{\tau}} l_{11}^{\tilde{t}} l_{12}^{\tilde{t}}+k_{11}^{\tilde{t}} k_{12}^{\tilde{t}} l_{1}^{\tilde{\nu}_{\tau}} l_{2}^{\tilde{\nu}_{\tau}}\right) m_{\tilde{\chi}_{1}^{+}} m_{\tilde{\chi}_{2}^{+}}\left(m_{\tilde{t}_{1}}^{2}+m_{\tilde{\nu}_{\tau}}^{2}-m_{b}^{2}-m_{\tau}^{2}\right) \\
& +2\left(k_{1}^{\tilde{\nu}_{\tau}} l_{2}^{\tilde{\nu}_{\tau}} l_{11}^{\tilde{t}} l_{12}^{\tilde{t}}+k_{2}^{\tilde{\nu}_{\tau}} k_{11}^{\tilde{t}} k_{12}^{\tilde{t}} l_{1}^{\tilde{\nu}_{\tau}}\right) m_{\tau} m_{\tilde{\chi}_{1}^{+}}\left(m_{\tilde{t}_{1}}^{2}-m_{b}^{2}\right) \\
& +2\left(k_{1}^{\tilde{\nu}_{\tau}} k_{11}^{\tilde{t}} k_{12}^{\tilde{t}} l_{2}^{\tilde{\nu}_{\tau}}+k_{2}^{\tilde{\nu}_{\tau}} l_{1}^{\tilde{\nu}_{\tau}} l_{11}^{\tilde{t}} l_{12}^{\tilde{t}}\right) m_{\tau} m_{\tilde{\chi}_{2}^{+}}\left(m_{\tilde{t}_{1}}^{2}-m_{b}^{2}\right) \\
& +2\left(k_{1}^{\tilde{\nu}_{\tau}} k_{2}^{\tilde{\nu}_{\tau}} k_{12}^{\tilde{t}} l_{11}^{\tilde{t}}+k_{11}^{\tilde{t}} l_{1}^{\tilde{\nu}_{\tau}} l_{2}^{\tilde{\nu}_{\tau}} l_{12}^{\tilde{t}}\right) m_{b} m_{\tilde{\chi}_{1}^{+}}\left(m_{\tilde{\nu}_{\tau}}^{2}-m_{\tau}^{2}\right) \\
& +2\left(k_{1}^{\tilde{\nu}_{\tau}} k_{2}^{\tilde{\nu}_{\tau}} k_{11}^{\tilde{t}} l_{12}^{\tilde{t}}+k_{12}^{\tilde{t}} l_{1}^{\tilde{\nu}_{\tau}} l_{2}^{\tilde{\nu}_{\tau}} l_{11}^{\tilde{t}}\right) m_{b} m_{\tilde{\chi}_{2}^{+}}\left(m_{\tilde{\nu}_{\tau}}^{2}-m_{\tau}^{2}\right) \\
& -4\left(k_{1}^{\tilde{\nu}_{\tau}} k_{12}^{\tilde{t}} l_{2}^{\tilde{\nu}_{\tau}} l_{11}^{\tilde{t}}+k_{2}^{\tilde{\nu}_{\tau}} k_{11}^{\tilde{t}} l_{1}^{\tilde{\nu}_{\tau}} l_{12}^{\tilde{t}}\right) m_{b} m_{\tau} m_{\tilde{\chi}_{1}^{+}} m_{\tilde{\chi}_{2}^{+}} \text {, }
\end{aligned}
$$




$$
\begin{aligned}
c_{33}= & \left(k_{1}^{\tilde{\nu}_{\tau}} k_{2}^{\tilde{\nu}_{\tau}} k_{11}^{\tilde{t}} k_{12}^{\tilde{t}}+l_{1}^{\tilde{\nu}_{\tau}} l_{2}^{\tilde{\nu}_{\tau}} l_{11}^{\tilde{t}} l_{12}^{\tilde{t}}\right)\left(m_{\tilde{t}_{1}}^{2}+m_{\tilde{\nu}_{\tau}}^{2}-m_{b}^{2}-m_{\tau}^{2}\right) \\
& -2\left(k_{1}^{\tilde{\nu}_{\tau}} k_{2}^{\tilde{\nu}_{\tau}} k_{12}^{\tilde{t}} l_{11}^{\tilde{t}}+k_{11}^{\tilde{t}} l_{1}^{\tilde{\nu}_{\tau}} l_{2}^{\tilde{\nu}_{\tau}} l_{12}^{\tilde{t}}\right) m_{b} m_{\tilde{\chi}_{1}^{+}}-2\left(k_{1}^{\tilde{\nu}_{\tau}} k_{2}^{\tilde{\nu}_{\tau}} k_{11}^{\tilde{t}} l_{12}^{\tilde{t}}+k_{12}^{\tilde{t}} l_{1}^{\tilde{\nu}_{\tau}} l_{2}^{\tilde{\nu}_{\tau}} l_{11}^{\tilde{t}}\right) m_{b} m_{\tilde{\chi}_{2}^{+}} \\
& -\left(k_{1}^{\tilde{\nu}_{\tau}} k_{2}^{\tilde{\nu}_{\tau}} l_{11}^{\tilde{t}} l_{12}^{\tilde{t}}+k_{11}^{\tilde{t}} k_{12}^{\tilde{t}} l_{1}^{\tilde{\nu}_{\tau}} l_{2}^{\tilde{\nu}_{\tau}}\right) m_{\tilde{\chi}_{1}^{+}} m_{\tilde{\chi}_{2}^{+}}-4\left(k_{1}^{\tilde{\nu}_{\tau}} k_{11}^{\tilde{t}} l_{2}^{\tilde{\nu}_{\tau}} l_{12}^{\tilde{t}}+k_{2}^{\tilde{\nu}_{\tau}} k_{12}^{\tilde{t}} l_{1}^{\tilde{\nu}_{\tau}} l_{11}^{\tilde{t}}\right) m_{b} m_{\tau} \\
& -2\left(k_{1}^{\tilde{\nu}_{\tau}} l_{2}^{\tilde{\nu}_{\tau}} l_{11}^{\tilde{t}} l_{12}^{\tilde{t}}+k_{2}^{\tilde{\nu}_{\tau}} k_{11}^{\tilde{t}} k_{12}^{\tilde{t}} l_{1}^{\tilde{\nu}_{\tau}}\right) m_{\tau} m_{\tilde{\chi}_{1}^{+}}-2\left(k_{1}^{\tilde{\nu}_{\tau}} k_{11}^{\tilde{t}} k_{12}^{\tilde{t}} \tilde{\nu}_{2}^{\tilde{\nu}_{\tau}}+k_{2}^{\tilde{\nu}_{\tau}} l_{1}^{\tilde{\nu}_{\tau}} l_{11}^{\tilde{t}} l_{12}^{\tilde{t}}\right) m_{\tau} m_{\tilde{\chi}_{2}^{+}} \\
c_{34}= & -\left(k_{1}^{\tilde{\nu}_{\tau}} k_{2}^{\tilde{\nu}_{\tau}} k_{11}^{\tilde{t}} k_{12}^{\tilde{t}}+l_{1}^{\tilde{\nu}_{\tau}} l_{2}^{\tilde{\nu}_{\tau}} l_{11}^{\tilde{t}} l_{12}^{\tilde{t}}\right), \\
c_{15}= & c_{25}=c_{35}=0 .
\end{aligned}
$$

The coefficients $c_{2 i}$ are obtained from $c_{1 i}$ by the replacements: $k_{11}^{\tilde{t}} \rightarrow k_{12}^{\tilde{t}}, l_{11}^{\tilde{t}} \rightarrow l_{12}^{\tilde{t}}, k_{1}^{\tilde{\nu}_{\tau}} \rightarrow$ $k_{2}^{\tilde{\nu}_{\tau}}, l_{1}^{\tilde{\nu}_{\tau}} \rightarrow l_{2}^{\tilde{\nu}_{\tau}}$ and $m_{\tilde{\chi}_{1}^{+}} \rightarrow m_{\tilde{\chi}_{2}^{+}}$. In the case of $\tilde{t}_{1} \rightarrow b \tilde{\tau}_{1}^{+} \nu_{\tau}$ one finds:

$$
\begin{aligned}
& W_{\nu_{\tau} \tilde{\tau}_{1}}(s)=\lambda^{\frac{1}{2}}\left(s, m_{\tilde{t}_{1}}^{2}, m_{b}^{2}\right), \\
& c_{11}=\frac{1}{2}\left(l_{11}^{\tilde{\tau}}\right)^{2}\left(l_{11}^{\tilde{t}}\right)^{2} m_{\tilde{\chi}_{1}^{+}}^{2} m_{\tilde{\tau}_{1}}^{4}\left(m_{\tilde{t}_{1}}^{2}-m_{b}^{2}\right), \\
& c_{12}=\left(l_{11}^{\tilde{\tau}}\right)^{2}\left[\left(l_{11}^{\tilde{t}}\right)^{2} m_{\tilde{\tau}_{1}}^{2} m_{\tilde{\chi}_{1}^{+}}^{2}\left(m_{b}^{2}-m_{\tilde{t}_{1}}^{2}-\frac{1}{2} m_{\tilde{\tau}_{1}}^{2}\right)+\frac{1}{2}\left(k_{11}^{\tilde{t}}\right)^{2} m_{\tilde{\tau}_{1}}^{4}\left(m_{\tilde{t}_{1}}^{2}-m_{b}^{2}\right)\right. \\
& \left.-2 k_{11}^{\tilde{t}} l_{11}^{\tilde{t}} m_{b} m_{\tilde{\chi}_{1}^{+}} m_{\tilde{\tau}_{1}}^{4}\right] \text {, } \\
& c_{13}=\left(l_{11}^{\tilde{\tau}}\right)^{2}\left[\frac{1}{2}\left(l_{11}^{\tilde{t}}\right)^{2} m_{\tilde{\chi}_{1}^{+}}^{2}\left(m_{\tilde{t}_{1}}^{2}+2 m_{\tilde{\tau}_{1}}^{2}-m_{b}^{2}\right)+4 k_{11}^{\tilde{t}} l_{11}^{\tilde{t}} m_{b} m_{\tilde{\chi}_{1}^{+}} m_{\tilde{\tau}_{1}}^{2}\right. \\
& \left.+\left(k_{11}^{\tilde{t}}\right)^{2} m_{\tilde{\tau}_{1}}^{2}\left(m_{b}^{2}-m_{\tilde{t}_{1}}^{2}-\frac{1}{2} m_{\tilde{\tau}_{1}}^{2}\right)\right], \\
& c_{14}=\left(l_{11}^{\tilde{\tau}}\right)^{2}\left[\frac{1}{2}\left(k_{11}^{\tilde{t}}\right)^{2}\left(2 m_{\tilde{\tau}_{1}}^{2}+m_{\tilde{t}_{1}}^{2}-m_{b}^{2}\right)-\frac{1}{2}\left(l_{11}^{\tilde{t}}\right)^{2} m_{\tilde{\chi}_{1}^{+}}^{2}-2 k_{11}^{\tilde{t}} l_{11}^{\tilde{t}} m_{b} m_{\tilde{\chi}_{1}^{+}}\right] \text {, } \\
& c_{15}=-\frac{1}{2}\left(l_{11}^{\tilde{\tau}}\right)^{2}\left(k_{11}^{\tilde{t}}\right)^{2} \\
& c_{31}=l_{11}^{\tilde{\tau}} l_{12}^{\tilde{\tau}} l_{11}^{\tilde{t}} l_{12}^{\tilde{t}} m_{\tilde{\chi}_{1}^{+}} m_{\tilde{\chi}_{2}^{+}} m_{\tilde{\tau}_{1}}^{4}\left(m_{\tilde{t}_{1}}^{2}-m_{b}^{2}\right), \\
& c_{32}=l_{11}^{\tilde{\tau}} l_{12}^{\tilde{\tau}}\left[k_{11}^{\tilde{t}} k_{12}^{\tilde{t}} m_{\tilde{\tau}_{1}}^{4}\left(m_{\tilde{t}_{1}}^{2}-m_{b}^{2}\right)+2 l_{11}^{\tilde{t}} l_{12}^{\tilde{t}} m_{\tilde{\chi}_{1}^{+}} m_{\tilde{\chi}_{2}^{+}} m_{\tilde{\tau}_{1}}^{2}\left(m_{b}^{2}-\frac{1}{2} m_{\tilde{\tau}_{1}}^{2}-m_{\tilde{t}_{1}}^{2}\right)\right. \\
& \left.-2 l_{11}^{\tilde{t}} k_{12}^{\tilde{t}} m_{b} m_{\tilde{\chi}_{1}^{+}} m_{\tilde{\tau}_{1}}^{4}-2 l_{12}^{\tilde{t}} k_{11}^{\tilde{t}} m_{b} m_{\tilde{\chi}_{2}^{+}} m_{\tilde{\tau}_{1}}^{4}\right], \\
& c_{33}=l_{11}^{\tilde{\tau}} l_{12}^{\tilde{\tau}}\left[l_{11}^{\tilde{t}} l_{12}^{\tilde{t}} m_{\tilde{\chi}_{1}^{+}} m_{\tilde{\chi}_{2}^{+}}\left(2 m_{\tilde{\tau}_{1}}^{2}+m_{\tilde{t}_{1}}^{2}-m_{b}^{2}\right)+4 l_{11}^{\tilde{t}} k_{12}^{\tilde{t}} m_{b} m_{\tilde{\chi}_{1}^{+}} m_{\tilde{\tau}_{1}}^{2}\right. \\
& \left.+4 l_{12}^{\tilde{t}} k_{11}^{\tilde{t}} m_{b} m_{\tilde{\chi}_{2}^{+}} m_{\tilde{\tau}_{1}}^{2}-2 k_{11}^{\tilde{t}} k_{12}^{\tilde{t}} m_{\tilde{\tau}_{1}}^{2}\left(m_{\tilde{t}_{1}}^{2}+\frac{1}{2} m_{\tilde{\tau}_{1}}^{2}-m_{b}^{2}\right)\right], \\
& c_{34}=l_{11}^{\tilde{\tau}} l_{12}^{\tilde{\tau}}\left[k_{11}^{\tilde{t}} k_{12}^{\tilde{t}}\left(2 m_{\tilde{\tau}_{1}}^{2}+m_{\tilde{t}_{1}}^{2}-m_{b}^{2}\right)-2 l_{11}^{\tilde{t}} k_{12}^{\tilde{t}} m_{b} m_{\tilde{\chi}_{1}^{+}}\right. \\
& \left.-2 l_{12}^{\tilde{t}} k_{11}^{\tilde{t}} m_{b} m_{\tilde{\chi}_{2}^{+}}-l_{11}^{\tilde{t}} l_{12}^{\tilde{t}} m_{\tilde{\chi}_{1}^{+}} m_{\tilde{\chi}_{2}^{+}}\right] \text {, } \\
& c_{35}=-l_{11}^{\tilde{\tau}} l_{12}^{\tilde{\tau}} k_{11}^{\tilde{t}} k_{12}^{\tilde{t}} .
\end{aligned}
$$


The coefficients $c_{2 i}$ are obtained from $c_{1 i}$ by the replacements: $k_{11}^{\tilde{t}} \rightarrow k_{12}^{\tilde{t}}, l_{11}^{\tilde{t}} \rightarrow l_{12}^{\tilde{t}}, l_{11}^{\tilde{\tau}} \rightarrow l_{12}^{\tilde{\tau}}$ and $m_{\tilde{\chi}_{1}^{+}} \rightarrow m_{\tilde{\chi}_{2}^{+}}$. To get the coefficients for $\tilde{t}_{1} \rightarrow b \tilde{\tau}_{2} \nu_{\tau}$ one has to make the replacements: $l_{1 i}^{\tilde{\tau}} \rightarrow l_{2 i}^{\tilde{\tau}}$ and $m_{\tilde{\tau}_{1}} \rightarrow m_{\tilde{\tau}_{2}}$. For $\tilde{t}_{1} \rightarrow b \tilde{e}_{L}^{+} \nu_{e}$ one gets the corresponding coefficients by the replacements: $l_{1 i}^{\tilde{\tau}} \rightarrow u_{1 i}$ and $m_{\tilde{\tau}_{1}} \rightarrow m_{\tilde{e}_{L}}$.

\section{APPENDIX B: COUPLINGS}

Here we give the couplings that were used in Eq. (13): The Yukawa couplings of the sfermions are given by:

$$
Y_{\tau}=\frac{m_{\tau}}{\sqrt{2} m_{W} \cos \beta}, \quad Y_{b}=\frac{m_{b}}{\sqrt{2} m_{W} \cos \beta}, \quad Y_{t}=\frac{m_{t}}{\sqrt{2} m_{W} \sin \beta} .
$$

The $\tilde{q}_{i}-q^{\prime}-\tilde{\chi}_{j}^{ \pm}$couplings read then

$$
l_{i j}^{\tilde{q}}=\mathcal{R}_{i n}^{\tilde{q}} \mathcal{O}_{j n}^{q}, \quad k_{i j}^{\tilde{q}}=\mathcal{R}_{i 1}^{\tilde{q}} \mathcal{O}_{j 2}^{q^{\prime}}
$$

with

$$
\mathcal{O}_{j}^{t}=\left(\begin{array}{c}
-V_{j 1} \\
Y_{t} V_{j 2}
\end{array}\right), \quad \mathcal{O}_{j}^{b}=\left(\begin{array}{c}
-U_{j 1} \\
Y_{b} U_{j 2}
\end{array}\right) .
$$

where $U_{i j}$ and $V_{i j}$ are the mixing matrices of the charginos [14]. In case of sleptons we have:

$$
l_{i j}^{\tilde{\tau}}=\mathcal{R}_{i n}^{\tilde{\tau}} \mathcal{O}_{j n}^{\tau}, \quad k_{i j}^{\tilde{\tau}}=0, \quad l_{j}^{\tilde{\nu}}=-V_{j 1}, \quad k_{j}^{\tilde{\nu}}=Y_{\tau} U_{j 2},
$$

with

$$
\mathcal{O}_{j}^{\tau}=\left(\begin{array}{c}
-U_{j 1} \\
Y_{\tau} U_{j 2}
\end{array}\right)
$$

The $\tilde{q}_{i}-q-\tilde{\chi}_{k}^{0}$ couplings are given by

$$
a_{i k}^{\tilde{q}}=\mathcal{R}_{i n}^{\tilde{q}} \mathcal{A}_{k n}^{f}, \quad b_{i k}^{\tilde{q}}=\mathcal{R}_{i n}^{\tilde{q}} \mathcal{B}_{k n}^{f}
$$

with

$$
\mathcal{A}_{k}^{f}=\left(\begin{array}{c}
f_{L k}^{f} \\
h_{R k}^{f}
\end{array}\right), \quad \mathcal{B}_{k}^{f}=\left(\begin{array}{c}
h_{L k}^{f} \\
f_{R k}^{f}
\end{array}\right),
$$

and

$$
\begin{aligned}
h_{L k}^{t} & =Y_{t}\left(\sin \beta N_{k 3}-\cos \beta N_{k 4}\right) \\
f_{L k}^{t} & =\frac{-2 \sqrt{2}}{3} \sin \theta_{W} N_{k 1}-\sqrt{2}\left(\frac{1}{2}-\frac{2}{3} \sin \theta_{W} q\right) \frac{N_{k 2}}{\cos \theta_{W}} \\
h_{R k}^{t} & =Y_{t}\left(\sin \beta N_{k 3}-\cos \beta N_{k 4}\right) \\
f_{R k}^{t} & =\frac{-2 \sqrt{2}}{3} \sin \theta_{W}\left(\tan \theta_{W} N_{k 2}-N_{k 1}\right) \\
h_{L k}^{b} & =-Y_{b}\left(\cos \beta N_{k 3}+\sin \beta N_{k 4}\right) \\
f_{L k}^{b} & =\frac{\sqrt{2}}{3} \sin \theta_{W} N_{k 1}+\sqrt{2}\left(\frac{1}{2}-\frac{1}{3} \sin \theta_{W} q\right) \frac{N_{k 2}}{\cos \theta_{W}} \\
h_{R k}^{b} & =-Y_{b}\left(\cos \beta N_{k 3}+\sin \beta N_{k 4}\right) \\
f_{R k}^{b} & =\frac{\sqrt{2}}{3} \sin \theta_{W}\left(\tan \theta_{W} N_{k 2}-N_{k 1}\right)
\end{aligned}
$$


where $N_{i j}$ is the mixing matrix of the neutralinos [15]. The couplings $\overline{\tilde{t}}_{i}-\tilde{b}_{j}-W^{+}$read

$$
A_{\tilde{t}_{i} \tilde{b}_{j}}^{W}=\left(A_{\tilde{b}_{i} \tilde{t}_{j}}^{W}\right)^{T}=\frac{1}{\sqrt{2}}\left(\begin{array}{rr}
\cos \theta_{\tilde{b}} \cos \theta_{\tilde{t}} & -\sin \theta_{\tilde{b}} \cos \theta_{\tilde{t}} \\
-\cos \theta_{\tilde{b}} \sin \theta_{\tilde{t}} & \sin \theta_{\tilde{b}} \sin \theta_{\tilde{t}}
\end{array}\right) .
$$

The couplings $\overline{\tilde{t}}_{i}-\tilde{b}_{j}-H^{+}$are given by

$$
\begin{aligned}
& C_{\tilde{t}_{i} \tilde{b}_{j}}^{H}=\left(C_{\tilde{b}_{i} \tilde{t}_{j}}^{H}\right)^{T} \\
& \quad=\frac{1}{\sqrt{2} m_{W}} \mathcal{R}^{\tilde{t}}\left(\begin{array}{cc}
m_{b}^{2} \tan \beta+m_{t}^{2} \cot \beta-m_{W}^{2} \sin (2 \beta) & m_{b}\left(A_{b} \tan \beta+\mu\right) \\
m_{t}\left(A_{t} \cot \beta+\mu\right) & 2 m_{b} m_{t} / \sin (2 \beta)
\end{array}\right)\left(\mathcal{R}^{\tilde{b}}\right)^{\dagger} .
\end{aligned}
$$

The $W^{+}{ }_{-} \tilde{\chi}_{j}^{-}-\tilde{\chi}_{k}^{0}$ couplings read:

$$
\begin{aligned}
& O_{k j}^{L \prime}=\frac{V_{j 2}}{\sqrt{2}}\left(\sin \beta N_{k 3}-\cos \beta N_{k 4}\right)+V_{j 1}\left(\sin \theta_{W} N_{k 1}+\cos \theta_{W} N_{k 2}\right) \\
& O_{k j}^{R \prime}=\frac{U_{j 2}}{\sqrt{2}}\left(\cos \beta N_{k 3}+\sin \beta N_{k 4}\right)+U_{j 1}\left(\sin \theta_{W} N_{k 1}+\cos \theta_{W} N_{k 2}\right) .
\end{aligned}
$$

The $H^{+}-\tilde{\chi}_{j}^{-}-\tilde{\chi}_{k}^{0}$ couplings are given by:

$$
\begin{aligned}
Q_{k j}^{L \prime}=\cos \beta[ & V_{j 1}\left(\cos \beta N_{k 4}-\sin \beta N_{k 3}\right) \\
& \left.+\frac{V_{j 2}}{\sqrt{2}}\left(2 \sin \theta_{W} N_{k 1}+\left(\cos \theta_{W}-\sin \theta_{W} \tan \theta_{W}\right) N_{k 2}\right)\right], \\
Q_{k j}^{R \prime}=\sin \beta[ & U_{j 1}\left(\cos \beta N_{k 3}+\sin \beta N_{k 4}\right) \\
& \left.\quad-\frac{U_{j 2}}{\sqrt{2}}\left(2 \sin \theta_{W} N_{k 1}+\left(\cos \theta_{W}-\sin \theta_{W} \tan \theta_{W}\right) N_{k 2}\right)\right] .
\end{aligned}
$$




\section{REFERENCES}

[1] For an introduction to supersymmetry, see J. Wess and J. Bagger, Supersymmetry and Supergravity (Princeton University Press, 1983); P. Fayet and S. Ferrara, Phys. Rep. 32, 249 (1977); H. P. Nilles, Phys. Rep. 110, 1 (1984).

[2] H. E. Haber and G.L. Kane, Phys. Rep. 117, 75 (1984).

[3] Proc. of the Workshop on Physics at LEP2, CERN 96-01, Vol. I, edited by G. Altarelli, T. Sjöstrand, and F. Zwirner; J. Amundson et al., Proceedings of the 1996 DPF/DPB Summer Study on High-Energy Physics, Snowmass, Colorado, 1996, edited by D.G. Cassel, L. Trindle Gennari, R.H. Siemann, p. 655; A. Bartl et al., ibid., p. 693; S. Mrenna et al., ibid., p. 681.

[4] M. Carena et al., hep-ex/9802006, hep-ex/9712022.

[5] V. Barger, M. S. Berger, J. F. Gunion, and T. Han, Phys. Rep. 286, 1 (1997).

[6] ECFA/DESY LC Physics Working Group, E. Accomando et al., Phys. Rep. 299, 1 (1998).

[7] X. Tata, hep-ph/9510287, Talk given at Theoretical Advanced Study Institute in Elementary Particle Physics (TASI 95): QCD and Beyond, Boulder, CO. In "Boulder 1995, QCD and beyond" 163-219.

[8] A. Bartl, W. Majerotto, and W. Porod, Z. Phys. C 64, 499 (1994); erratum ibid. C 68, 518 (1995); A. Bartl, H. Eberl, S. Kraml, W. Majerotto, and W. Porod, Z. Phys. C 73, 469 (1997); A. Bartl, H. Eberl, S. Kraml, W. Majerotto, W. Porod, and A. Sopczak, Z. Phys. C 76, 49 (1997).

[9] J. Ellis and S. Rudaz, Phys. Lett. 128B, 248 (1983); G. Altarelli and R. Rückl, Phys. Lett. 144B, 126 (1984); I. Bigi and S. Rudaz, Phys. Lett. 153B, 335 (1985).

[10] K. I. Hikasa and M. Kobayashi, Phys. Rev. D 36, 724 (1987).

[11] W. Porod and T. Wöhrmann, Phys. Rev. D 55, 2907 (1997).

[12] Aleph Coll., Phys. Lett. B412, 173 (1997); Delphi Coll., Eur. Phys. J. C2, 1 (1998); Opal Coll., Eur. Phys. J. C5, 19 (1998).

[13] A. Brignole, Phys. Lett. B277, 313 (1992).

[14] A. Bartl, H. Fraas, W. Majerotto, and B. Mößlacher, Z. Phys. C 55, 257 (1992).

[15] A. Bartl, H. Fraas, W. Majerotto, and N. Oshimo, Phys. Rev. D 40, 1594 (1989). 


\section{TABLES}

\begin{tabular}{llll}
\hline \hline Input: & $\tan \beta=3$ & $\mu=530 \mathrm{GeV}$ & $M=270 \mathrm{GeV}$ \\
& $M_{\tilde{D}}=370 \mathrm{GeV}$ & $M_{\tilde{Q}}=340 \mathrm{GeV}$ & $A_{b}=150 \mathrm{GeV}$ \\
& $M_{\tilde{E}}=210 \mathrm{GeV}$ & $M_{\tilde{L}}=210 \mathrm{GeV}$ & $A_{\tau}=150 \mathrm{GeV}$ \\
& $m_{\tilde{t}_{1}}=250 \mathrm{GeV}$ & $\cos \theta_{\tilde{t}}=0.6$ & \\
Calculated & $m_{\tilde{\chi}_{1}^{0}}=130$ & $m_{\tilde{\chi}_{1}^{+}}=253$ & $m_{\tilde{\chi}_{2}^{+}}=550$ \\
& $m_{\tilde{b}_{1}}=342 \mathrm{GeV}$ & $m_{\tilde{b}_{2}}=372 \mathrm{GeV}$ & $\cos \theta_{\tilde{b}}=0.98$ \\
& $m_{\tilde{\tau}_{1}}=209 \mathrm{GeV}$ & $m_{\tilde{\tau}_{2}}=217 \mathrm{GeV}$ & $\cos \theta_{\tilde{\tau}}=0.68$ \\
& $m_{\tilde{e}_{L}}=213 \mathrm{GeV}$ & $m_{\tilde{\nu}_{e}}=m_{\tilde{\nu}_{\tau}}=204 \mathrm{GeV}$ & \\
\hline \hline
\end{tabular}

TABLE I. Input parameters and resulting quantities used in Fig. 2 and 3.

\begin{tabular}{llll}
\hline \hline Input: & $\tan \beta=3$ & $\mu=750 \mathrm{GeV}$ & $M=380 \mathrm{GeV}$ \\
& $M_{\tilde{D}}=550 \mathrm{GeV}$ & $M_{\tilde{Q}}=500 \mathrm{GeV}$ & $A_{b}=400 \mathrm{GeV}$ \\
& $M_{\tilde{E}}=275 \mathrm{GeV}$ & $M_{\tilde{L}}=275 \mathrm{GeV}$ & $A_{\tau}=400 \mathrm{GeV}$ \\
& $m_{\tilde{t}_{1}}=350 \mathrm{GeV}$ & $\cos \theta_{\tilde{t}}=0.7$ & $m_{A^{0}}=110 \mathrm{GeV}$ \\
Calculated & $m_{\tilde{\chi}_{1}^{0}}=186$ & $m_{\tilde{\chi}_{1}^{+}}=368$ & $m_{\tilde{\chi}_{2}^{+}}=764$ \\
& $m_{\tilde{b}_{1}}=502 \mathrm{GeV}$ & $m_{\tilde{b}_{2}}=551 \mathrm{GeV}$ & $\cos \theta_{\tilde{b}}=0.99$ \\
& $m_{\tilde{\tau}_{1}}=274 \mathrm{GeV}$ & $m_{\tilde{\tau}_{2}}=281 \mathrm{GeV}$ & $\cos \theta_{\tilde{\tau}}=0.69$ \\
& $m_{\tilde{e}_{L}}=278 \mathrm{GeV}$ & $m_{\tilde{\nu}_{e}}=m_{\tilde{\nu}_{\tau}}=270 \mathrm{GeV}$ & $m_{H^{+}}=136 \mathrm{GeV}$ \\
\hline \hline
\end{tabular}

TABLE II. Input parameters and resulting quantities used in Fig. 4 and 5. 


\section{FIGURES}
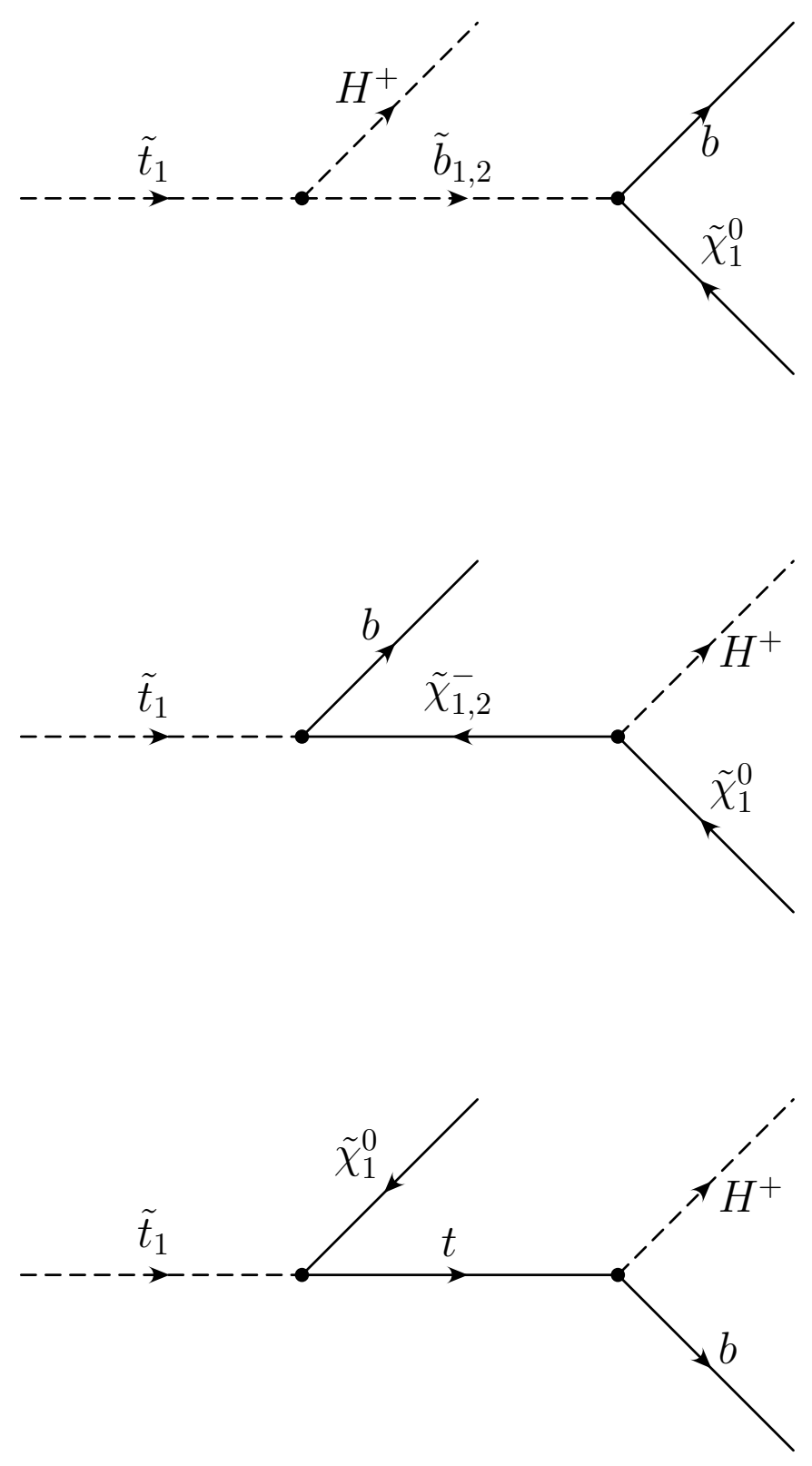

FIG. 1. Feynman diagrams for the decay $\tilde{t}_{1} \rightarrow H^{+} b \tilde{\chi}_{1}^{0}$. 


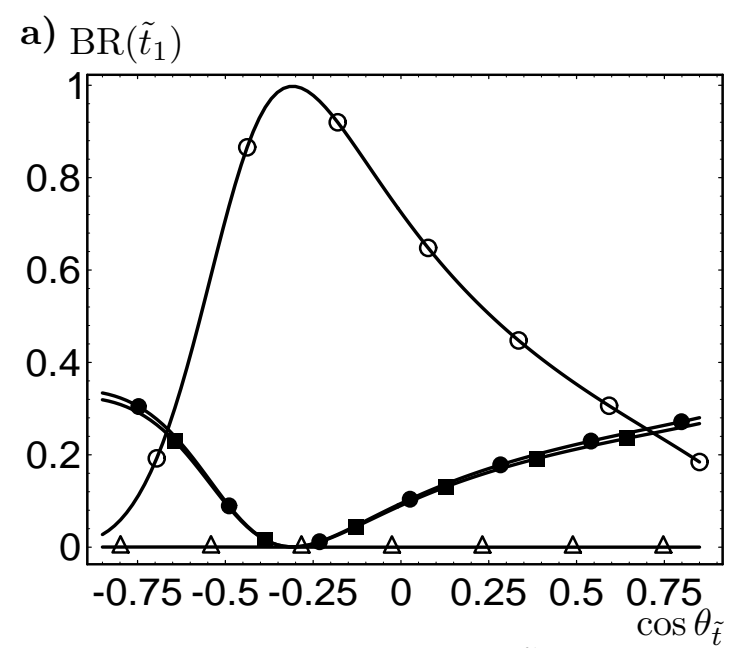

b) $\operatorname{BR}\left(\tilde{t}_{1}\right)$

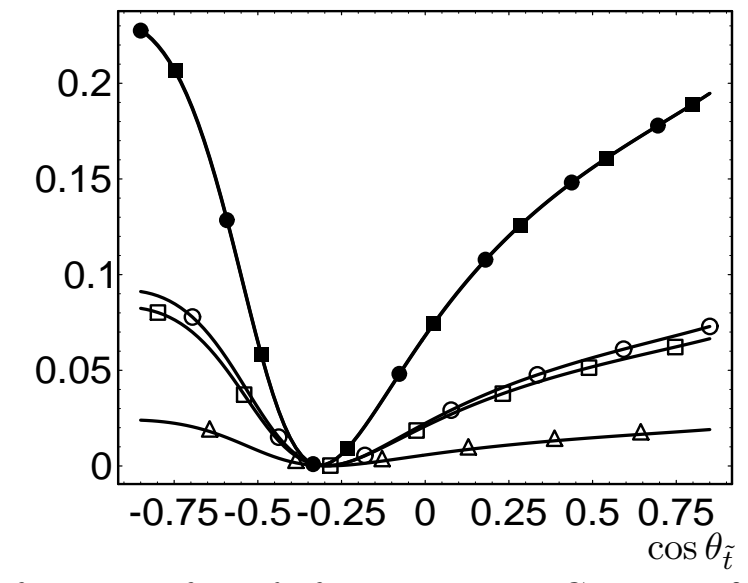

FIG. 2. Branching ratios for $\tilde{t}_{1}$ decays as a function of $\cos \theta_{\tilde{t}}$ for $m_{\tilde{t}_{1}}=250 \mathrm{GeV}, \tan \beta=3$, $\mu=530 \mathrm{GeV}$, and $M=270 \mathrm{GeV}$. The other parameters are given in Table I. The curves in a) correspond to the transitions: $\circ \tilde{t}_{1} \rightarrow b W^{+} \tilde{\chi}_{1}^{0}, \triangle \tilde{t}_{1} \rightarrow c \tilde{\chi}_{1}^{0}, \mathbf{\square}\left(\tilde{t}_{1} \rightarrow b e^{+} \tilde{\nu}_{e}\right)+\left(\tilde{t}_{1} \rightarrow b \nu_{e} \tilde{e}_{L}^{+}\right)$, and - $\left(\tilde{t}_{1} \rightarrow b \tau^{+} \tilde{\nu}_{\tau}\right)+\left(\tilde{t}_{1} \rightarrow b \nu_{\tau} \tilde{\tau}_{1}\right)+\left(\tilde{t}_{1} \rightarrow b \nu_{\tau} \tilde{\tau}_{2}\right)$. The curves in b) correspond to the transitions: $\circ \tilde{t}_{1} \rightarrow b \nu_{e} \tilde{e}_{L}^{+}, \square \tilde{t}_{1} \rightarrow b \nu_{\tau} \tilde{\tau}_{1}, \triangle \tilde{t}_{1} \rightarrow b \nu_{\tau} \tilde{\tau}_{2}, \mathbf{\square} \tilde{t}_{1} \rightarrow b e^{+} \tilde{\nu}_{e}$, and $\bullet \tilde{t}_{1} \rightarrow b \tau^{+} \tilde{\nu}_{\tau}$.
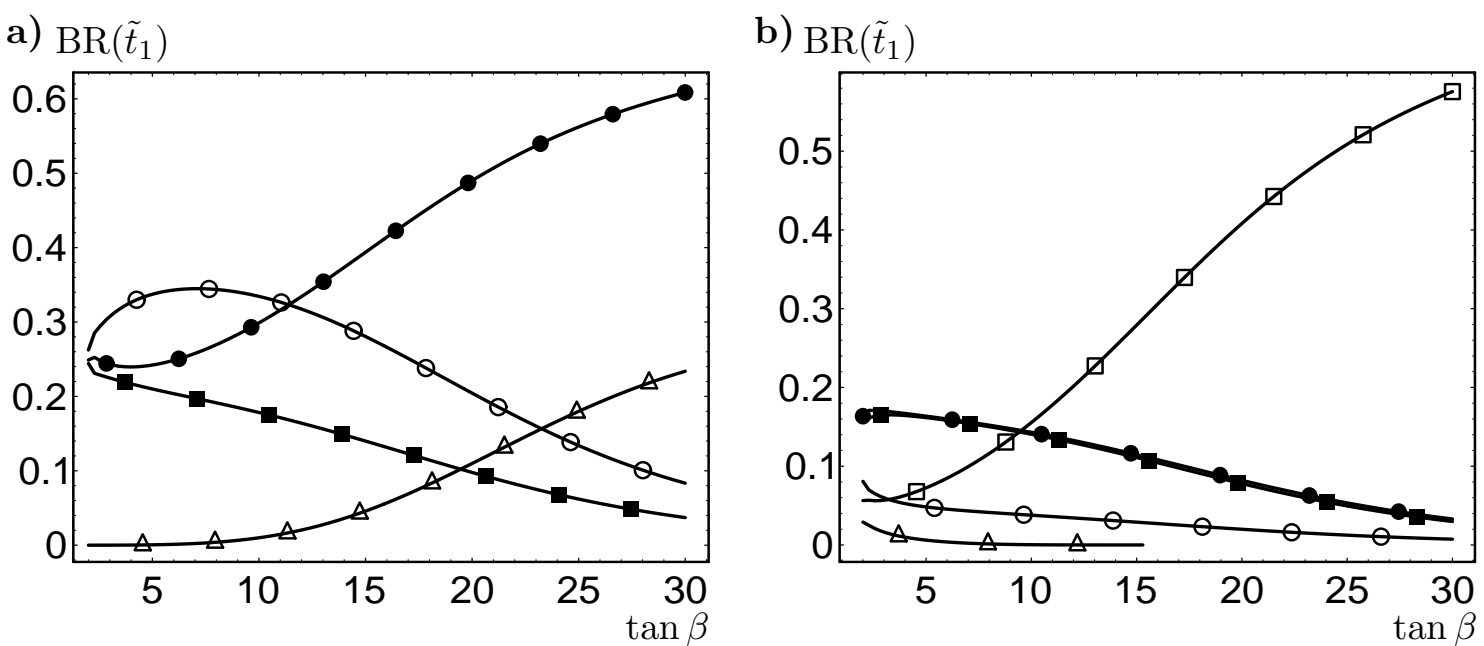

FIG. 3. Branching ratios for $\tilde{t}_{1}$ decays as a function of $\tan \beta$ for $m_{\tilde{t}_{1}}=250 \mathrm{GeV}, \cos \theta_{\tilde{t}}=0.6$, $\mu=530 \mathrm{GeV}, M=270 \mathrm{GeV}$. The other parameters are given in Table I. The curves in a) correspond to the transitions: $\circ \tilde{t}_{1} \rightarrow b W^{+} \tilde{\chi}_{1}^{0}, \triangle \tilde{t}_{1} \rightarrow c \tilde{\chi}_{1}^{0}, \mathbf{\square}\left(\tilde{t}_{1} \rightarrow b e^{+} \tilde{\nu}_{e}\right)+\left(\tilde{t}_{1} \rightarrow b \nu_{e} \tilde{e}_{L}^{+}\right)$, and $\bullet\left(\tilde{t}_{1} \rightarrow b \tau^{+} \tilde{\nu}_{\tau}\right)+\left(\tilde{t}_{1} \rightarrow b \nu_{\tau} \tilde{\tau}_{1}\right)+\left(\tilde{t}_{1} \rightarrow b \nu_{\tau} \tilde{\tau}_{2}\right)$. The curves in b) correspond to the transitions: $\circ \tilde{t}_{1} \rightarrow b \nu_{e} \tilde{e}_{L}^{+}, \square \tilde{t}_{1} \rightarrow b \nu_{\tau} \tilde{\tau}_{1}, \triangle \tilde{t}_{1} \rightarrow b \nu_{\tau} \tilde{\tau}_{2}, \boldsymbol{\square} \tilde{t}_{1} \rightarrow b e^{+} \tilde{\nu}_{e}$, and $\bullet \tilde{t}_{1} \rightarrow b \tau^{+} \tilde{\nu}_{\tau}$. 


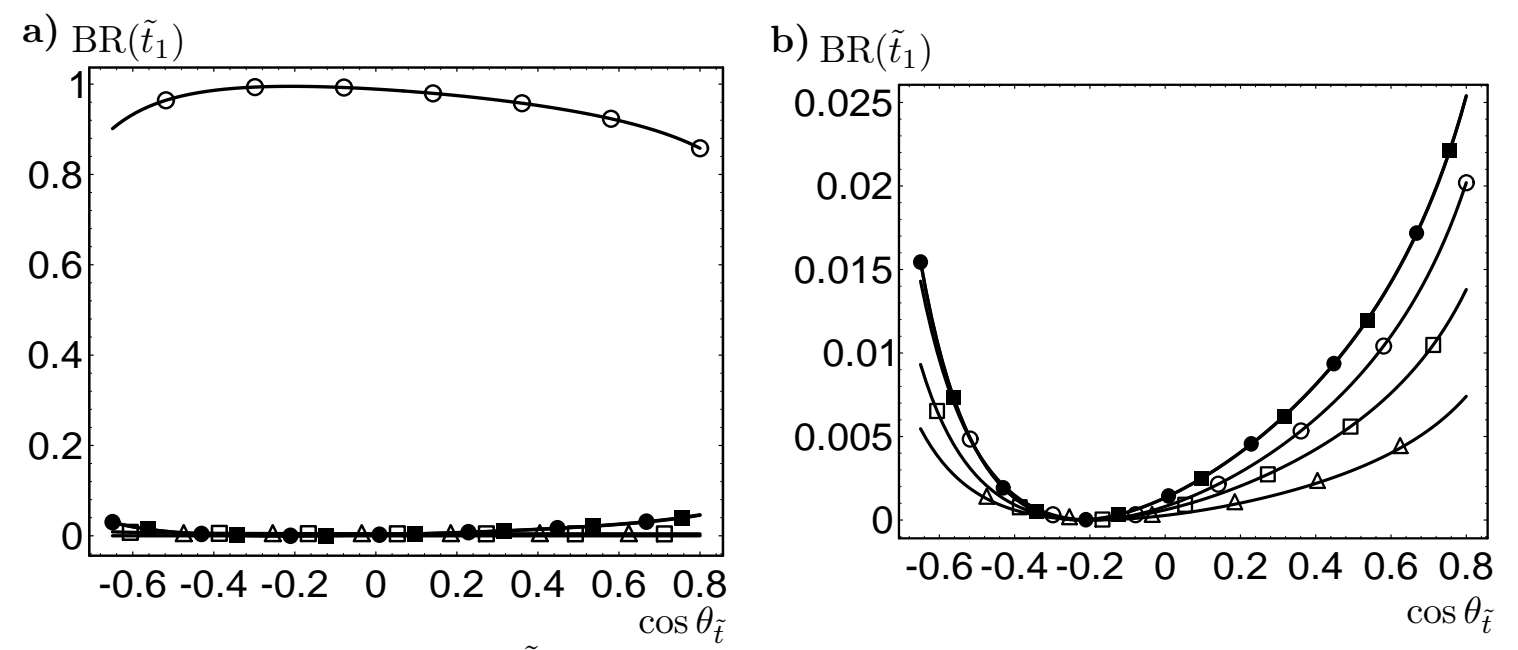

FIG. 4. Branching ratios for $\tilde{t}_{1}$ decays as a function of $\cos \theta_{\tilde{t}}$ for $m_{\tilde{t}_{1}}=350 \mathrm{GeV}, \tan \beta=3$, $\mu=750 \mathrm{GeV}, M=380 \mathrm{GeV}$, and $m_{A^{0}}=110 \mathrm{GeV}$. The other parameters are given in Table II. The curves in a) correspond to the transitions: $\circ \tilde{t}_{1} \rightarrow b W^{+} \tilde{\chi}_{1}^{0}, \square \tilde{t}_{1} \rightarrow b H^{+} \tilde{\chi}_{1}^{0}, \triangle \tilde{t}_{1} \rightarrow c \tilde{\chi}_{1}^{0}$, $\mathbf{\square}\left(\tilde{t}_{1} \rightarrow b e^{+} \tilde{\nu}_{e}\right)+\left(\tilde{t}_{1} \rightarrow b \nu_{e} \tilde{e}_{L}^{+}\right)$, and $\bullet\left(\tilde{t}_{1} \rightarrow b \tau^{+} \tilde{\nu}_{\tau}\right)+\left(\tilde{t}_{1} \rightarrow b \nu_{\tau} \tilde{\tau}_{1}\right)+\left(\tilde{t}_{1} \rightarrow b \nu_{\tau} \tilde{\tau}_{2}\right)$. The curves in b) correspond to the transitions: $\circ \tilde{t}_{1} \rightarrow b \nu_{e} \tilde{e}_{L}^{+}, \square \tilde{t}_{1} \rightarrow b \nu_{\tau} \tilde{\tau}_{1}, \triangle \tilde{t}_{1} \rightarrow b \nu_{\tau} \tilde{\tau}_{2}, \mathbf{\square} \tilde{t}_{1} \rightarrow b e^{+} \tilde{\nu}_{e}$, and $\bullet \tilde{t}_{1} \rightarrow b \tau^{+} \tilde{\nu}_{\tau}$.
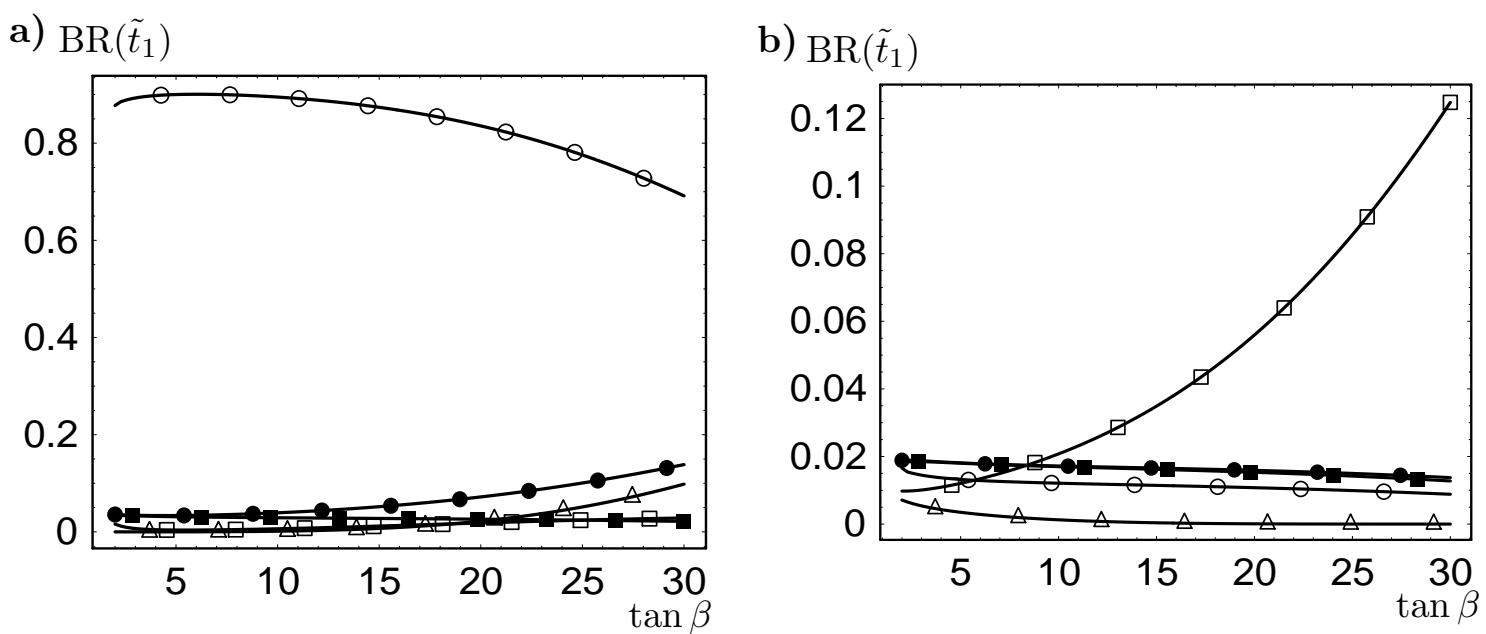

FIG. 5. Branching ratios for $\tilde{t}_{1}$ decays as a function of $\tan \beta$ for $m_{\tilde{t}_{1}}=350 \mathrm{GeV}, \cos \theta_{\tilde{t}}=0.7$, $\mu=750 \mathrm{GeV}, M=380 \mathrm{GeV}$ and $m_{A^{0}}=110 \mathrm{GeV}$. The other parameters are given in Table II. The curves in a) correspond to the transitions: $\circ \tilde{t}_{1} \rightarrow b W^{+} \tilde{\chi}_{1}^{0}, \square \tilde{t}_{1} \rightarrow b H^{+} \tilde{\chi}_{1}^{0}, \triangle \tilde{t}_{1} \rightarrow c \tilde{\chi}_{1}^{0}$, $\mathbf{\square}\left(\tilde{t}_{1} \rightarrow b e^{+} \tilde{\nu}_{e}\right)+\left(\tilde{t}_{1} \rightarrow b \nu_{e} \tilde{e}_{L}^{+}\right)$, and $\bullet\left(\tilde{t}_{1} \rightarrow b \tau^{+} \tilde{\nu}_{\tau}\right)+\left(\tilde{t}_{1} \rightarrow b \nu_{\tau} \tilde{\tau}_{1}\right)+\left(\tilde{t}_{1} \rightarrow b \nu_{\tau} \tilde{\tau}_{2}\right)$. The curves in b) correspond to the transitions: $\circ \tilde{t}_{1} \rightarrow b \nu_{e} \tilde{e}_{L}^{+}, \square \tilde{t}_{1} \rightarrow b \nu_{\tau} \tilde{\tau}_{1}, \triangle \tilde{t}_{1} \rightarrow b \nu_{\tau} \tilde{\tau}_{2}, \mathbf{\square} \tilde{t}_{1} \rightarrow b e^{+} \tilde{\nu}_{e}$, and $\bullet \tilde{t}_{1} \rightarrow b \tau^{+} \tilde{\nu}_{\tau}$. 


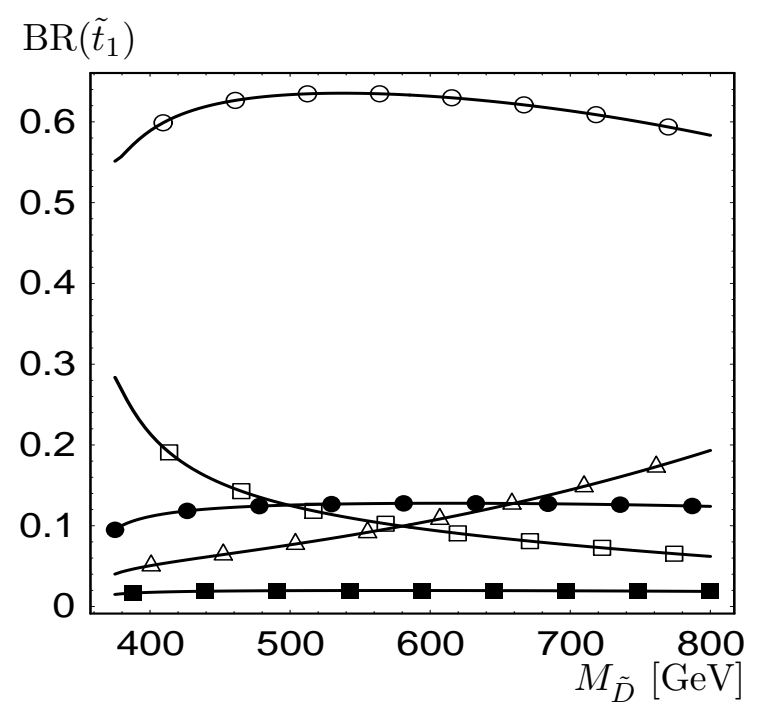

FIG. 6. Branching ratios for $\tilde{t}_{1}$ decays as a function of $M_{\tilde{D}}$ for $m_{\tilde{t}_{1}}=350 \mathrm{GeV}, \cos \theta_{\tilde{t}}=0.7$, $\tan \beta=30, \mu=750 \mathrm{GeV}$, and $m_{A^{0}}=90 \mathrm{GeV}$. The other input parameters are the same as in Table II. The curves in a) correspond to the transitions: $\circ \tilde{t}_{1} \rightarrow b W^{+} \tilde{\chi}_{1}^{0}, \square \tilde{t}_{1} \rightarrow b H^{+} \tilde{\chi}_{1}^{0}$, $\triangle \tilde{t}_{1} \rightarrow c \tilde{\chi}_{1}^{0}, \mathbf{\square}\left(\tilde{t}_{1} \rightarrow b e^{+} \tilde{\nu}_{e}\right)+\left(\tilde{t}_{1} \rightarrow b \nu_{e} \tilde{e}_{L}^{+}\right)$, and $\bullet\left(\tilde{t}_{1} \rightarrow b \tau^{+} \tilde{\nu}_{\tau}\right)+\left(\tilde{t}_{1} \rightarrow b \nu_{\tau} \tilde{\tau}_{1}\right)+\left(\tilde{t}_{1} \rightarrow b \nu_{\tau} \tilde{\tau}_{2}\right)$. 